\title{
Consensus of cyber-physical power systems based on multi-agent systems with communication constraints
}

\author{
Chi HUANG ${ }^{1,2}$, , Chengli FENG $^{3}$, Jinde CAO $^{2}$
}

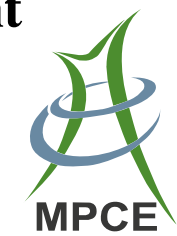

\begin{abstract}
The consensus protocol of cyber-physical power systems is proposed based on fractional-order multi-agent systems with communication constraints. It aims to enable each generator to reach a time-varying common rotor angle and rotor speed. Communication constraints including event-triggered sampling and partial information transmission are considered to render the consensus protocol more realistic. The Zeno behavior is excluded during the system sampling process. A sufficient condition is derived to solve the consensus problem. The effectiveness of the proposed consensus protocol is demonstrated by a numerical example.
\end{abstract}

Keywords Cyber-physical power system, Fractional-order system, Multi-agent system, Communication constraint

CrossCheck date: 17 August 2018

Received: 21 December 2017/ Accepted: 17 August 2018/Published online: 17 January 2019

(C) The Author(s) 2019

$\triangle$ Chi HUANG

huangchi@swufe.edu.cn

Chengli FENG

fengchengli666@163.com

Jinde CAO

jdcao@seu.edu.cn

1 School of Economic Information Engineering, Southwestern University of Finance and Economics, Chengdu 611130, China

2 School of Mathematics, Southeast University, Nanjing 210096, China

3 College of Mathematics, Taiyuan University of Technology, Taiyuan 030024, China

\section{Introduction}

The concept of cyber-physical power systems (CPPSs) is first proposed in [1] as a dedicated case of a cyberphysical system in a power system. It is composed of a large number of computing devices (servers, computers, and embedded computing devices), data acquisition devices (sensors, phasor measurement unit, and embedded data acquisition equipment), and physical devices (large-scale generator set, distributed power supply, and load). These devices are connected through communication and transmission networks. CPPSs combine the advantages of cyber-physical systems and power systems. However, it also experiences many technical and security challenges $[2,3]$. Owing to the ability to collect and handle massive information compared with traditional power systems, studies on CPPSs such as traffic networks [4] and aviation electric power systems [5] have been performed. Among these, the stability of CPPSs has been emphasized, because an unstable power supply may lead to voltage collapse and breakdown of the entire power system [6, 7]. Most studies on stability in the existing literature are focused on the supply-demand balance and rotor angle stability. Specifically, the target of the supply-demand balance is to continuously match the production and consumption of electricity across networks [8]. The rotor angle stability [9] enables the CPPS to operate smoothly and efficiently by allowing each generator to reach a time-varying common rotor angle and speed, separately. Both of them can be regarded as a consensus perspective. Thus, many studies use the consensus theory to solve stability problems in CPPSs [10, 11].

A multi-agent system (MAS) model has been extensively studied as an effective method for the consensus problem $[12,13]$. It is a cooperative system whereby agents 
can share their information to communicate with each other. The MAS has many significant real-world applications, such as sensor networks [14], unmanned air vehicles formations [15, 16], and microgrids [17]. Furthermore, many studies have applied the MAS to a CPPS [18-21]. In [18], a distributed multi-agent-based load shedding algorithm that can yield an efficient load shedding decision based on the discovered global information was proposed. In [20], an innovative approach was proposed to use realtime scheduling techniques for the automation of electric loads in CPPSs. In [21], a distributed control method to enhance power system rotor angle stability based on the second-order MAS consensus was proposed, where each generator can be regarded as an agent to communicate with each other. Nevertheless, most of the studies on CPPSs are based on integer-order MASs. In fact, real-world processes are generally (or most likely) fractional-order systems [22, 23]. Many physical systems exhibit fractional dynamical behavior owing to their special materials and chemical properties [24-27]. Thus, it is highly desirable to study CPPSs with fractional-order MASs.

Communication constraints that are crucial factors to the system performance cannot be ignored in practice. Among them, the partial information transmission is significant. That is to say, not all of the information can be transmitted to their neighbors perfectly [28-30]. Energy saving is another important topic. Considering the massive communicating and computing work in CPPSs, sampling would be an efficient method to reduce resources. Traditional periodic sampling techniques would typically waste much energy and consequently shorten the lifespan of the system to a certain degree [31]. To address this problem, event-triggered control has attracted significant attention from research into the consensus of MASs [32-37]. The Zeno behavior must be excluded in the event sampling process; otherwise, continuous communication will be required again [38]. However, among the existing literatures, the proofs absent of the Zeno behavior are not presented (or are naturally avoided), because events can only occur at sampling time instants that are a multiple of some given sampling period $[34,39]$. Therefore, it is necessary to propose a more specific proof when the Zeno behavior is excluded.

Our current study is of practical significance because it can effectively reduce the gap between the physical and cyber worlds. However, few results have been found in the existing literature. Motivated by the discussions above, we herein consider the consensus of CPPSs based on fractional-order MASs with communication constraints. Our objective is to ensure that each generator achieves a timevarying common rotor angle and rotor speed. The contribution of this paper can be summarized in three aspects: 1) a fractional-order MAS modeling with communication constraints is established for the consensus of the CPPS; 2) based on communication constraints, a distributed event condition is introduced for energy conservation, and consensus criteria are obtained that can be applied to more practical systems; 3) a finite number of broadcasts and updates exist by each agent in any finite time period, thus ensuring that the Zeno behavior does not occur in the CPPS. The effectiveness of the proposed consensus criteria is illustrated with a numerical example.

\section{Model description and preliminaries}

In this section, some basic notions and properties of the algebraic graph theory are introduced.

Each agent can be abstracted as a node. Let $\mathcal{G}=$ $(\mathcal{V}, \mathcal{E}, G)$ be a weighted directed network of order $N$, with the set of nodes (vertices) $\mathcal{V}=\{1,2, \ldots, N\}$, the set of directed edges $\mathcal{E} \subseteq \mathcal{V} \times \mathcal{V}$, and a weighted adjacency matrix $\boldsymbol{A}=\left(a_{i j}\right)_{N \times N}$. A directed edge in graph $\mathcal{G}$ is denoted by the ordered pairs of nodes $\mathcal{E}_{i j}=(i, j)$, where $i$ and $j$ are called the terminal and initial nodes, respectively. If edge $(j, i) \in \mathcal{E}$, then node $j$ is called a neighbor of node $i$, implying that node $i$ can receive information from node $j$. Therefore, $a_{i j}>0$; otherwise, $a_{i j}=0$. The neighborhood index set of node $i$ is denoted by $\mathcal{N}_{i}=\{j \in \mathcal{V} \mid(j, i) \in \mathcal{E}\}$, while we indicate with $\left|\mathcal{N}_{i}\right|$ its cardinality (the number of neighbors of node $i$ ). $\|\cdot\|$ and $\|\cdot\|_{1}$ represent the Euclidean norm and one norm for a vector or a matrix, respectively. $\boldsymbol{I}_{n}$ and $\boldsymbol{O}_{n}$ express the identity matrix and zero matrix with dimension $n$, respectively. $D_{+}^{\alpha}$ represents the fractional-order Dini(upper right) derivative. Matrix $\boldsymbol{A}>\mathbf{0}$ denotes that $\boldsymbol{A}$ is a positively defined matrix, and $\operatorname{diag}\{\cdots\}$ denotes a diagonal matrix.

\subsection{Fractional calculus and preliminary lemmas}

Definition 1 The fractional integral with non-integer order $\alpha>0$ of function $p(\cdot)$ is defined as follows:

$D_{t_{0}, t}^{-\alpha} p(t)=\frac{1}{\Gamma(\alpha)} \int_{t_{0}}^{t}(t-\tau)^{\alpha-1} p(\tau) \mathrm{d} \tau$

where $\Gamma(\cdot)$ is the gamma function $\Gamma(t)=\int_{0}^{\infty} t^{\alpha-1} e^{-t} \mathrm{~d} t$.

Several definitions exist that refer to the fractional derivative of order $\alpha>0$. Compared with other definitions, the Caputo derivative has a unique advantage; it only claims the initial conditions provided as the integer-order derivative of $p(\cdot)$ and the function itself. Therefore, our consideration is a fractional-order MAS with a Caputo derivative, whose definition is given below. 
Definition 2 The Caputo derivative of fractional order $\alpha>0$ of function $p(\cdot)$ is defined as follows:

$$
\begin{aligned}
{ }_{t_{0}}^{C} D_{t}^{\alpha} p(t) & =D_{t_{0}, t}^{-(n-\alpha)} p^{(n)}(t) \\
& =\frac{1}{\Gamma(n-\alpha)} \int_{t_{0}}^{t}(t-\tau)^{n-\alpha-1} p^{(n)}(\tau) \mathrm{d} \tau
\end{aligned}
$$

where $n-1<\alpha \leq n \in \mathbf{Z}^{+}$.

For simplicity, $D_{0, t}^{-\alpha},{ }_{0}^{C} D_{t}^{\alpha}$ are rewritten as $D^{-\alpha}$ and $D^{\alpha}$, respectively. Some properties of these two operators are introduced.

Lemma 1 [40] If $p(t), g(t) \in C^{n}[0,+\infty)$ and $n-$ $1<\alpha, \beta \leq n \in \mathbf{Z}^{+}$, then:

1) $D^{-\alpha} D^{-\beta} p(t)=D^{-(\alpha+\beta)} p(t), \alpha, \beta>0$.

2) $D^{\alpha} D^{-\alpha} p(t)=p(t), n=1$.

3) $D^{-\alpha} D^{\alpha} p(t)=p(t)-\sum_{j=0}^{n-1}\left(t^{j} / j !\right) p^{(j)}(0), \alpha \geq 0$.

4) $D^{\alpha}(p(t) \pm g(t))=D^{\alpha} p(t) \pm D^{\alpha} g(t)$.

where $C^{n}(M, N)$ denotes the space consisting of $n$-order continuous differentiable functions from $M$ into $N$.

Lemma 2 [25] Let $\boldsymbol{x}(t)=\left(x_{1}(t), x_{2}(t), \ldots, x_{n}(t)\right)^{\mathrm{T}} \in \mathbf{R}^{n}$ be a differential vector-value function. Then, for any time instant $t \geq t_{0}$,

${ }_{t_{0}}^{C} D_{t}^{\alpha}\left[\boldsymbol{x}^{\mathrm{T}}(t) \boldsymbol{P} \boldsymbol{x}(t)\right] \leq \boldsymbol{x}^{\mathrm{T}}(t) \boldsymbol{P}_{t_{0}}^{C} D_{t}^{\alpha} \boldsymbol{x}(t)+\left({ }_{t_{0}}^{C} D_{t}^{\alpha} \boldsymbol{x}^{\mathrm{T}}(t)\right) \boldsymbol{P} \boldsymbol{x}(t)$

Therefore,

${ }_{t_{0}}^{C} D_{t}^{\alpha}\left[\boldsymbol{x}^{\mathrm{T}}(t) \boldsymbol{P} \boldsymbol{x}(t)\right] \leq 2 \boldsymbol{x}^{\mathrm{T}}(t) \boldsymbol{P}_{t_{0}}^{C} D_{t}^{\alpha} \boldsymbol{x}(t)$

where $\boldsymbol{P} \in \mathbf{R}^{n \times n}$ is a symmetric positive definite matrix, $\alpha \in(0,1)$.

\subsection{Network model}

In this section, we will discuss the stability of the rotor angle. The CPPS can be modeled by the formulas as follows [41].

$\dot{\delta}_{i}=\omega_{i}$

$\left\{\begin{array}{l}\dot{\omega}_{i}=-\frac{D_{i}}{M_{i}} \omega_{i}+\frac{1}{M_{i}}\left(P_{m i}-P_{e i}\right) \\ \dot{\boldsymbol{E}}_{i}^{\prime}=\boldsymbol{F}_{i} \boldsymbol{E}_{i}^{\prime}+\boldsymbol{L}_{i}\left(\delta_{i}\right) \boldsymbol{V}_{i}+\boldsymbol{H}_{i} \boldsymbol{E}_{f i} \\ P_{e i}=\boldsymbol{V}_{i}^{\mathrm{T}}\left[\boldsymbol{R}_{i}\left(\delta_{i}\right) \boldsymbol{V}_{i}+\boldsymbol{T}_{i}\left(\delta_{i}\right) \boldsymbol{E}_{i}^{\prime}\right] \\ P_{m i}=\frac{B_{m i} p}{A_{m i} p} u_{m i} \\ E_{f i}=\frac{B_{f i} p}{A_{f i} p} u_{f i} \\ \boldsymbol{V}=[\boldsymbol{Y}-\boldsymbol{R}(\boldsymbol{\delta})]^{-1} \boldsymbol{T}(\boldsymbol{\delta}) \boldsymbol{E}^{\prime}\end{array}\right.$ where $\delta_{i}$ is the rotor angle; $\omega_{i}$ is the rotor speed; $D_{i}$ is the damping coefficient; $M_{i}$ is the inertia constant; $P_{m i}$ is the mechanical power; $P_{e i}$ is the electrical power; $\boldsymbol{E}_{i}^{\prime}$ is the internal bus voltage vector; $\boldsymbol{V}$ is the generator terminal voltage vector. $\boldsymbol{R}(\boldsymbol{\delta})$ and $\boldsymbol{T}(\boldsymbol{\delta})$ are matrices comprised of elements $\boldsymbol{R}_{i}\left(\delta_{i}\right)$ and $\boldsymbol{T}_{i}\left(\delta_{i}\right)$, respectively. $\boldsymbol{R}_{i}\left(\delta_{i}\right), \boldsymbol{T}_{i}\left(\delta_{i}\right) \boldsymbol{F}_{i}$, and $\boldsymbol{H}_{i}$ are descried in [41] in detail.

A classical coherency criterion states that a generator pair $(i, j)$ is considered coherent if

$\left\{\begin{array}{l}\lim _{t \rightarrow \infty}\left\|\delta_{i}-\delta_{j}\right\|=0 \\ \lim _{t \rightarrow \infty}\left\|\omega_{i}-\omega_{j}\right\|=0\end{array}\right.$

which can be considered a consensus problem of a secondorder multi-agent system. It appears that we can use a multi-agent system to study the rotor angle stability of the CPPS.

From (1) and (2), if the controller input is chosen in the following form:

$\tilde{P}_{m i}=M_{i} u_{i}^{\prime}+D_{i} \omega_{i}+P_{e i}$

where $u_{i}^{\prime}$ is the new input to be determined, then the differential equations of the rotor angle and rotor speed in the CPPS is described as follows:

$\left\{\begin{array}{l}\dot{\delta}_{i}=\omega_{i} \\ \dot{\omega}_{i}=u_{i}^{\prime}\end{array}\right.$

Thus, the design of $u_{i}^{\prime}$ is important.

Next, we discuss the rotor angle stability of the CPPS with a multi-agent system model.

The fractional-order calculation is more general, because it is an expansion of the integer-order calculation. We consider the fractional-order multi-agent system as follows:

$$
\left\{\begin{array}{l}
D^{\beta} \boldsymbol{x}_{i}(t)=\boldsymbol{v}_{i}(t) \\
D^{\beta} \boldsymbol{v}_{i}(t)=\boldsymbol{u}_{i}(t) \quad i=1,2, \ldots, N
\end{array}\right.
$$

where $\beta \in(0,1] ; \boldsymbol{x}_{i}(t) \in \mathbf{R}^{n}, \boldsymbol{v}_{i}(t) \in \mathbf{R}^{n}$ are the position and speed information of the $i^{\text {th }}$ agent, respectively; $\boldsymbol{u}_{i}(t)$ is the control input.

To achieve a common position and speed for the MAS based on the fractional-order MAS (6), we consider the following control protocol:

$$
\begin{aligned}
\boldsymbol{u}_{i}(t)= & \boldsymbol{f}\left(\boldsymbol{x}_{i}(t), \boldsymbol{v}_{i}(t), t\right)-\sum_{j=1, j \neq i}^{N} a_{i j}\left[\boldsymbol{H}_{i j}\left(\boldsymbol{x}_{i}(t)-\boldsymbol{x}_{j}(t)\right)\right. \\
& \left.+\boldsymbol{H}_{i j}\left(\boldsymbol{v}_{i}(t)-\boldsymbol{v}_{j}(t)\right)\right]
\end{aligned}
$$

where $\boldsymbol{A}=\left[a_{i j}\right]_{N \times N}$ is the weighted adjacency matrix, which represents the connection among agents. Considering the factors of the MAS and the influence of the external 
environment, the nonlinear term [42] is added to the control model of the system. $f: \mathbf{R}^{n} \times \mathbf{R}^{n} \times \mathbf{R}^{+} \rightarrow \mathbf{R}^{n}$ is a continuously differentiable vector-valued nonlinear function. $\boldsymbol{H}_{i j}=\operatorname{diag}\left\{h_{i j}^{1}, h_{i j}^{2}, \ldots, h_{i j}^{n}\right\} h_{i j}^{k}=0$ or 1 , for each $k=$ $1,2, \ldots, n$. It is called the channel matrix [28], owing to the imperfect signal transmission in a communications network.

Let $\quad \boldsymbol{R}_{i j}=a_{i j} \boldsymbol{H}_{i j}=\operatorname{diag}\left\{r_{i j}^{1}, r_{i j}^{2}, \cdots, r_{i j}^{n}\right\}, \quad$ for $\quad i, j=$ $1,2, \ldots, N$ and $\boldsymbol{R}_{i i}=-\sum_{j=1, j \neq i}^{N} \boldsymbol{R}_{i j}$.

The control input of the partial information transmission for the consensus of the MAS (7) can be rewritten in the following form:

$$
\begin{aligned}
\boldsymbol{u}_{i}(t)= & \boldsymbol{f}\left(\boldsymbol{x}_{i}(t), \boldsymbol{v}_{i}(t), t\right)-\sum_{j=1, j \neq i}^{N} \boldsymbol{R}_{i j}\left[\left(\boldsymbol{x}_{i}(t)-\boldsymbol{x}_{j}(t)\right)\right. \\
& \left.+\left(\boldsymbol{v}_{i}(t)-\boldsymbol{v}_{j}(t)\right)\right]
\end{aligned}
$$

for any $i, j=1,2, \ldots, N$. For mathematical derivation, we define the channel Laplacian matrix as follows:

$$
\boldsymbol{R}_{k}=\left[\begin{array}{cccc}
r_{11}^{k} & r_{12}^{k} & \ldots & r_{1 N}^{k} \\
r_{21}^{k} & r_{22}^{k} & \ldots & r_{2 N}^{k} \\
\vdots & \vdots & & \vdots \\
r_{N 1}^{k} & r_{N 2}^{k} & \ldots & r_{N N}^{k}
\end{array}\right] \quad k \in\{1,2, \ldots, n\}
$$

It is clear that the matrices $\boldsymbol{R}_{k}$ are decomposed from $\boldsymbol{R}_{i j}$. Moreover, $R_{k}(i, j)$ is the $k^{\text {th }}$ diagonal element of $\boldsymbol{R}_{i j}$ for any $i, j=1,2, \ldots, N$. Obviously, $\boldsymbol{R}_{k}$ has the zero row-sum property. Herein, we always assume that $\boldsymbol{R}_{k}$ is irreducible. Suppose $\boldsymbol{\xi}_{k}=\left[\xi_{k 1}, \xi_{k 2}, \ldots, \xi_{k N}\right]^{\mathrm{T}} \in \mathbf{R}^{N}$ satisfying $\sum_{i=1}^{N} \xi_{k i}=1$ is the normalized left eigenvector of matrix $\boldsymbol{R}_{k}$ corresponding to the eigenvalue 0 . From [43], $\xi_{k i}>0$ holds for all $i \in\{1,2, \ldots, N\}$ and $k \in\{1,2, \ldots, n\}$. Setting $\boldsymbol{\Phi}_{i}=$ $\operatorname{diag}\left\{\xi_{1 i}, \xi_{2 i}, \ldots, \xi_{n i}\right\}$, we can obtain $\sum_{i=1}^{N} \boldsymbol{\Phi}_{i} \boldsymbol{R}_{i j}=\mathbf{0}$.

Owing to the massive distributed components and the large number of data in the CPPS, energy saving appears particularly important. Compared with traditional period sampling, the event-trigger sampling strategy is more advantageous. Therefore, we adopt the event-trigger sampling method. The control input $\boldsymbol{u}_{i}(t)$ is converted into

$$
\left\{\begin{aligned}
\boldsymbol{u}_{i}(t)= & \boldsymbol{f}\left(\boldsymbol{x}_{i}(t), \boldsymbol{v}_{i}(t), t\right)-\sum_{j=1, j \neq i}^{N}\left[\boldsymbol{R}_{i j}\left(\boldsymbol{x}_{i}\left(t_{k}^{i}\right)-\boldsymbol{x}_{j}\left(t_{k^{\prime}}^{j}\right)\right)\right. \\
& \left.+\boldsymbol{R}_{i j}\left(\boldsymbol{v}_{i}\left(t_{k}^{i}\right)-\boldsymbol{v}_{j}\left(t_{k^{\prime}}^{j}\right)\right)\right] \quad t \in\left[t_{k}^{i}, t_{k+1}^{i}\right) \\
k^{\prime}(t)= & \underset{l \in N, t>t_{l}^{j}}{\arg \min }\left\{t-t_{l}^{j}\right\}
\end{aligned}\right.
$$

where $t_{k}^{i}$ is the sampling instant that is analytically determined by the following individual event condition:

$$
t_{k+1}^{i}=\inf \left\{t>t_{k}^{i}, E_{i}\left(\boldsymbol{x}\left(t_{k}^{i}\right), \boldsymbol{v}\left(t_{k}^{i}\right), \boldsymbol{x}_{i}(t), \boldsymbol{v}_{i}(t)\right)>0\right\}
$$

where

$$
\begin{aligned}
& E_{i}\left(\boldsymbol{x}\left(t_{k}^{i}\right), \boldsymbol{v}\left(t_{k}^{i}\right), \boldsymbol{x}_{i}(t), \boldsymbol{v}_{i}(t)\right)=\left\|\boldsymbol{e}_{x i}(t)\right\|_{1}+\left\|\boldsymbol{e}_{v i}(t)\right\|_{1} \\
& \quad-\varphi_{1} \sum_{j \in N_{i}}\left[\left\|\boldsymbol{R}_{i j}\left(\boldsymbol{x}_{i}\left(t_{k}^{i}\right)-\boldsymbol{x}_{j}\left(t_{k^{\prime}(t)}^{j}\right)\right)\right\|_{1}\right. \\
& \left.\quad+\left\|\boldsymbol{R}_{i j}\left(\boldsymbol{v}_{i}\left(t_{k}^{i}\right)-\boldsymbol{v}_{j}\left(t_{k^{\prime}(t)}^{j}\right)\right)\right\|_{1}\right]-\varphi_{2} e^{-\gamma\left(t-t_{0}\right)}, \\
& \boldsymbol{e}_{x i}(t)=\boldsymbol{x}_{i}\left(t_{k}^{i}\right)-\boldsymbol{x}_{i}(t), \boldsymbol{e}_{v i}(t)=\boldsymbol{v}_{i}\left(t_{k}^{i}\right)-\boldsymbol{v}_{i}(t), \\
& t \in\left[t_{k}^{i}, t_{k+1}^{i}\right), i=1,2, \ldots, N
\end{aligned}
$$

are the position measurement errors and speed measurement errors.

The following assumption, definitions, and lemmas will be used to derive our primary results. Thus, they are given as below:

Assumption 1 Assume that two positive constants $l_{1}, l_{2}$, exist such that the vector-valued nonlinear function $f(\cdot)$ satisfies

$$
\left\|\boldsymbol{f}\left(\boldsymbol{x}_{1}, \boldsymbol{x}_{2}, t\right)-\boldsymbol{f}\left(\boldsymbol{y}_{1}, \boldsymbol{y}_{2}, t\right)\right\| \leq \sum_{i=1}^{2} l_{i}\left\|\boldsymbol{x}_{i}-\boldsymbol{y}_{i}\right\|
$$

where $\boldsymbol{x}_{i}, \boldsymbol{y}_{i} \in \mathbf{R}^{n} ; t \geq 0$.

Definition 3 The consensus in MAS (6) is said to be achieved, if for any initial states,

$$
\begin{aligned}
& \lim _{t \rightarrow \infty}\left\|\boldsymbol{x}_{i}(t)-\boldsymbol{x}_{j}(t)\right\|=0 \\
& \lim _{t \rightarrow \infty}\left\|\boldsymbol{v}_{i}(t)-\boldsymbol{v}_{j}(t)\right\|=0
\end{aligned}
$$

Definition 4 System (9) does not exhibit the Zeno behavior, if $\inf _{k}\left\{t_{k+1}^{i}-t_{k}^{i}\right\}>0$, for all $i$. In other words, there is no system trajectory with an infinite number of events in a finite time.

Definition 5 [44] The two parameters form of the Mittag-Leffler function is defined as follows:

$$
E_{\alpha, \beta}(z)=\sum_{k=0}^{\infty} \frac{z^{k}}{\Gamma(\alpha k+\beta)}
$$

Lemma 3 [45] For two vectors $\boldsymbol{x}(t)$ and $\boldsymbol{y}(t) \in \mathbf{R}^{N n}$,

$$
\begin{aligned}
\boldsymbol{x}(t) & =\left[\boldsymbol{x}_{1}^{\mathrm{T}}(t), \boldsymbol{x}_{2}^{\mathrm{T}}(t), \ldots, \boldsymbol{x}_{N}^{\mathrm{T}}(t)\right]^{\mathrm{T}} \\
\boldsymbol{y}(t) & =\left[\boldsymbol{y}_{1}^{\mathrm{T}}(t), \boldsymbol{y}_{2}^{\mathrm{T}}(t), \ldots, \boldsymbol{y}_{N}^{\mathrm{T}}(t)\right]^{\mathrm{T}}
\end{aligned}
$$

we have the following properties: 
1) $\sum_{i=1}^{N}\left\|\boldsymbol{x}_{i}(t)\right\|_{1}=\|\boldsymbol{x}(t)\|_{1}$.

2) $\sum_{i=1}^{N} \sum_{j=1}^{N}\left\|\boldsymbol{x}_{i}(t)\right\|_{1}\left\|\boldsymbol{y}_{i}(t)\right\|_{1}=\|\boldsymbol{x}(t)\|_{1}\|\boldsymbol{y}(t)\|_{1}$.

3) $\|\boldsymbol{x}(t)\|_{1}\|\boldsymbol{x}(t)\|_{1} \leq N n\|\boldsymbol{x}(t)\|^{2}=N n \boldsymbol{x}^{\mathrm{T}}(t) \boldsymbol{x}(t)$.

Lemma 4 (Schur complement) The following linear matrix inequality

$$
\left[\begin{array}{ll}
\mathcal{Q}(x) & \mathcal{S}(x) \\
\mathcal{S}^{\mathrm{T}}(x) & \mathcal{R}(x)
\end{array}\right]>\mathbf{0}
$$

where $\mathcal{Q}(x)=\mathcal{Q}^{\mathrm{T}}(x)$ and $\mathcal{R}(x)=\mathcal{R}^{\mathrm{T}}(x)$ is equivalent to one of the following conditions.

1) $\mathcal{Q}(x)>\mathbf{0}, \boldsymbol{R}(x)-\mathcal{S}^{\mathrm{T}}(x) \mathcal{Q}(x)^{-1} \mathcal{S}(x)>\mathbf{0}$.

2) $\mathcal{R}(x)>\mathbf{0}, \mathcal{Q}(x)-\mathcal{S}(x) \mathcal{R}(x)^{-1} \mathcal{S}^{\mathrm{T}}(x)>\mathbf{0}$.

Lemma 5 [46] The Mittag-Leffler function generalizes the simple exponential function (recovered for $\beta=1$ ) and, if $0<\beta<1$, it interpolates the positive real axis to a stretched exponential and a power law according to

$$
E_{\beta}\left[-\left(\tau / \tau_{0}\right)^{\beta}\right] \sim \begin{cases}\exp \left[-\left(\tau / \tau_{0}\right)^{\beta} / \Gamma(1+\beta)\right] & \tau / \tau_{0} \rightarrow 0^{+} \\ \left(\tau / \tau_{0}\right)^{-\beta} / \Gamma(1-\beta) & \tau / \tau_{0} \rightarrow \infty\end{cases}
$$

\subsection{Primary results}

In this section, we shall obtain the consensus condition of the fractional-order MAS with communication constraints.

Let

$$
\begin{array}{ll}
\overline{\boldsymbol{x}}(t)=\sum_{k=1}^{N} \boldsymbol{\Phi}_{k} \boldsymbol{x}_{k}(t), & \overline{\boldsymbol{v}}(t)=\sum_{k=1}^{N} \boldsymbol{\Phi}_{k} \boldsymbol{v}_{k}(t) \\
\tilde{\boldsymbol{x}}_{i}(t)=\boldsymbol{x}_{i}(t)-\overline{\boldsymbol{x}}(t), & \tilde{\boldsymbol{v}}_{i}(t)=\boldsymbol{v}_{i}(t)-\overline{\boldsymbol{v}}(t)
\end{array}
$$

where $\overline{\boldsymbol{x}}(t)$ is the time-varying common position and $\overline{\boldsymbol{v}}(t)$ is the time-varying common speed for each agent; $\tilde{\boldsymbol{x}}(t), \tilde{\boldsymbol{v}}(t)$ are the errors between the current state information and the common state information. Subsequently, the consensus problem can be translated into a stable issue.

Because

$$
\tilde{\boldsymbol{x}}_{i}(t)=\boldsymbol{x}_{i}(t)-\sum_{k=1}^{N} \boldsymbol{\Phi}_{k} \boldsymbol{x}_{k}(t), \tilde{\boldsymbol{v}}_{i}(t)=\boldsymbol{v}_{i}(t)-\sum_{k=1}^{N} \boldsymbol{\Phi}_{k} \boldsymbol{v}_{k}(t)
$$

we can obtain

$$
\begin{aligned}
\sum_{i=1}^{N} \tilde{\boldsymbol{x}}_{i}^{\mathrm{T}}(t) \boldsymbol{\Phi}_{i} & =\sum_{i=1}^{N}\left(\boldsymbol{x}_{i}^{\mathrm{T}}(t)-\sum_{k=1}^{N} \boldsymbol{x}_{k}^{\mathrm{T}}(t) \boldsymbol{\Phi}_{k}\right) \boldsymbol{\Phi}_{i} \\
& =\sum_{i=1}^{N} \boldsymbol{x}_{i}^{\mathrm{T}}(t) \boldsymbol{\Phi}_{i}-\sum_{i=1}^{N} \sum_{k=1}^{N} \boldsymbol{x}_{k}^{\mathrm{T}}(t) \boldsymbol{\Phi}_{k} \boldsymbol{\Phi}_{i} \\
\sum_{i=1}^{N} \tilde{\boldsymbol{v}}_{i}^{\mathrm{T}}(t) \boldsymbol{\Phi}_{i} & =\sum_{i=1}^{N}\left(\boldsymbol{v}_{i}^{\mathrm{T}}(t)-\sum_{k=1}^{N} \boldsymbol{v}_{k}^{\mathrm{T}}(t) \boldsymbol{\Phi}_{k}\right) \boldsymbol{\Phi}_{i} \\
& =\sum_{i=1}^{N} \boldsymbol{v}_{i}^{\mathrm{T}}(t) \boldsymbol{\Phi}_{i}-\sum_{i=1}^{N} \sum_{k=1}^{N} \boldsymbol{v}_{k}^{\mathrm{T}}(t) \boldsymbol{\Phi}_{k} \boldsymbol{\Phi}_{i} .
\end{aligned}
$$

From $\sum_{i=1}^{N} \boldsymbol{\Phi}_{i}=\boldsymbol{I}_{n}$, we obtain

$$
\sum_{i=1}^{N} \tilde{\boldsymbol{x}}_{i}^{\mathrm{T}}(t) \boldsymbol{\Phi}_{i}=\mathbf{0}, \quad \sum_{i=1}^{N} \tilde{\boldsymbol{v}}_{i}^{\mathrm{T}}(t) \boldsymbol{\Phi}_{i}=\mathbf{0}
$$

Subsequently, we can obtain the following error dynamical system

$$
\left\{\begin{aligned}
D^{\beta} \tilde{\boldsymbol{x}}_{i}(t)= & \tilde{\boldsymbol{v}}_{i}(t) \\
D^{\beta} \tilde{\boldsymbol{v}}_{i}(t)= & \boldsymbol{u}_{i}(t)-\sum_{k=1}^{N} \boldsymbol{\Phi}_{k} \boldsymbol{u}_{k}(t) \\
= & \boldsymbol{f}\left(\boldsymbol{x}_{i}(t), \boldsymbol{v}_{i}(t), t\right)-\sum_{k=1}^{N} \boldsymbol{\Phi}_{k} \boldsymbol{f}\left(\boldsymbol{x}_{k}(t), \boldsymbol{v}_{k}(t), t\right) \\
& +\sum_{j=1}^{N} \boldsymbol{R}_{i j} \boldsymbol{e}_{x j}(t)+\sum_{j=1}^{N} \boldsymbol{R}_{i j} \tilde{\boldsymbol{x}}_{j}(t) \\
& +\sum_{j=1}^{N} \boldsymbol{R}_{i j} \boldsymbol{e}_{v j}(t)+\sum_{j=1}^{N} \boldsymbol{R}_{i j} \tilde{\boldsymbol{v}}_{j}(t)
\end{aligned}\right.
$$

Based on the analysis above, we can obtain the condition that the agents cooperate to achieve the time-varying common position and speed in the MAS.

Theorem 1 Suppose that Assumption 1 holds and $t_{0}^{i}=$ $t_{0}=0, i=1,2, \ldots, N$ under the event condition. $\boldsymbol{R}_{k}$ is irreducible. Therefore, the consensus in network (6) is achieved under the designed control input (9) if the scalar $\alpha>0$ exists that satisfies the following LMIs:

$$
\begin{aligned}
& \boldsymbol{Q}_{k}^{\mathrm{T}}\left(\boldsymbol{\Xi}_{k} \boldsymbol{R}_{k}+\boldsymbol{R}_{k}^{\mathrm{T}} \boldsymbol{\Xi}_{k}+\alpha \boldsymbol{I}_{N}\right) \boldsymbol{Q}_{k}<\mathbf{0} \\
& \mu_{1} \boldsymbol{I}_{n}-\left(l_{1}^{2}+l_{1} l_{2}+\frac{1}{2}\right) \boldsymbol{\Phi}_{i}>\mathbf{0} \\
& \mu_{2} \boldsymbol{I}_{n}-\left(l_{2}^{2}+l_{1} l_{2}+\frac{3}{2}\right) \boldsymbol{\Phi}_{i}>\mathbf{0} \\
& \alpha \boldsymbol{I}_{n}-\boldsymbol{\Phi}_{i}>\mathbf{0}
\end{aligned}
$$


where

$$
\begin{aligned}
\mu_{1} & =\frac{\alpha}{2}-2 M \phi_{1} N n-\frac{M^{2} \phi_{2}^{2} N n}{4 \kappa^{2}}, \\
\mu_{2} & =\frac{\alpha}{2}-2 M \phi_{1} N n-\frac{M^{2} \phi_{2}^{2} N n}{4 \sigma^{2}}, \\
\boldsymbol{\Xi}_{k} & =\operatorname{diag}\left\{\xi_{k 1}, \xi_{k 2}, \ldots, \xi_{k N}\right\}, \\
\boldsymbol{Q}_{k} & =\left[\begin{array}{l}
\xi_{k 1} \\
\xi_{k N}
\end{array}-\frac{\xi_{k 2}}{\xi_{k N}} \quad \ldots \quad-\frac{\xi_{k N-1}}{\xi_{k N}}\right], \\
M & =\max _{1 \leq i, j, p, q \leq N}\left(\boldsymbol{\Pi}_{i j}\right)_{p q}, \quad \phi_{1}=\frac{\varphi_{1} N_{M} N \bar{r}}{1-\varphi_{1} N_{M} N \bar{r}}, \\
\phi_{2} & =\frac{\varphi_{2} N}{1-\varphi_{1} N_{M} N \bar{r}}, \quad 0 \leq \varphi_{1}<\frac{1}{N_{M} N \bar{r}}, \quad \varphi_{2} \geq 0 .
\end{aligned}
$$

Proof Consider the following Lyapunov function candidate:

$V(t)=\frac{1}{2} \sum_{i=1}^{N}\left(\tilde{\boldsymbol{x}}_{i}^{\mathrm{T}}(t), \tilde{\boldsymbol{v}}_{i}^{\mathrm{T}}(t)\right)\left[\begin{array}{cc}\alpha \boldsymbol{I}_{n} & \boldsymbol{\Phi}_{i} \\ \boldsymbol{\Phi}_{i} & \boldsymbol{\Phi}_{i}\end{array}\right]\left[\begin{array}{c}\tilde{\boldsymbol{x}}_{i}(t) \\ \tilde{\boldsymbol{v}}_{i}(t)\end{array}\right]$

Let $\boldsymbol{y}_{i}^{\mathrm{T}}(t)=\left(\tilde{\boldsymbol{x}}_{i}^{\mathrm{T}}(t), \tilde{\boldsymbol{v}}_{i}^{\mathrm{T}}(t)\right)^{\mathrm{T}}$, then

$$
V(t)=\frac{1}{2} \sum_{i=1}^{N} \boldsymbol{y}_{i}^{\mathrm{T}}(t)\left[\begin{array}{cc}
\alpha \boldsymbol{I}_{n} & \boldsymbol{\Phi}_{i} \\
\boldsymbol{\Phi}_{i} & \boldsymbol{\Phi}_{i}
\end{array}\right] \boldsymbol{y}_{i}(t)
$$

By Lemma 4, $\left[\begin{array}{ll}\alpha \boldsymbol{I}_{n} & \boldsymbol{\Phi}_{i} \\ \boldsymbol{\Phi}_{i} & \boldsymbol{\Phi}_{i}\end{array}\right]>\mathbf{0}$ is equivalent to $\boldsymbol{\Phi}_{i}>\mathbf{0}$ and $\alpha \boldsymbol{I}_{n}-\boldsymbol{\Phi}_{i}>\mathbf{0}$. Then, $V(t) \geq 0 . V(t)=0$ if and only if $\boldsymbol{y}(t)=\mathbf{0}$. By calculating the time derivative of the $V(t)$ along the trajectory of the error system (10), the following equation can be obtained:

$$
\begin{aligned}
D^{\beta} V(t) & \leq \sum_{i=1}^{N} \boldsymbol{y}_{i}^{\mathrm{T}}(t)\left[\begin{array}{cc}
\alpha \boldsymbol{I}_{n} & \boldsymbol{\Phi}_{i} \\
\boldsymbol{\Phi}_{i} & \boldsymbol{\Phi}_{i}
\end{array}\right] D^{\alpha} \boldsymbol{y}_{i}(t) \\
= & \sum_{i=1}^{N}\left(\tilde{\boldsymbol{x}}_{i}^{\mathrm{T}}(t), \tilde{\boldsymbol{v}}_{i}^{\mathrm{T}}(t)\right)\left[\begin{array}{cc}
\alpha \boldsymbol{I}_{n} & \boldsymbol{\Phi}_{i} \\
\boldsymbol{\Phi}_{i} & \boldsymbol{\Phi}_{i}
\end{array}\right]\left[\begin{array}{c}
\tilde{\boldsymbol{v}}_{i}(t) \\
\boldsymbol{u}_{i}(t)-\sum_{k=1}^{N} \boldsymbol{\Phi}_{k} \boldsymbol{u}_{k}(t)
\end{array}\right] \\
= & \alpha \sum_{i=1}^{N} \tilde{\boldsymbol{x}}_{i}^{\mathrm{T}}(t) \tilde{\boldsymbol{v}}_{i}(t)+\sum_{i=1}^{N} \tilde{\boldsymbol{v}}_{i}^{\mathrm{T}}(t) \boldsymbol{\Phi}_{i} \tilde{\boldsymbol{v}}_{i}(t)+\sum_{i=1}^{N} \tilde{\boldsymbol{x}}_{i}^{\mathrm{T}}(t) \boldsymbol{\Phi}_{i} \\
& \cdot\left[\boldsymbol{f}\left(\boldsymbol{x}_{i}(t), \boldsymbol{v}_{i}(t), t\right)-\boldsymbol{f}(\overline{\boldsymbol{x}}(t), \overline{\boldsymbol{v}}(t), t)\right] \\
& +\sum_{i=1}^{N} \tilde{\boldsymbol{v}}_{i}^{\mathrm{T}}(t) \boldsymbol{\Phi}_{i}\left[\boldsymbol{f}\left(\boldsymbol{x}_{i}(t), \boldsymbol{v}_{i}(t), t\right)-\boldsymbol{f}(\overline{\boldsymbol{x}}(t), \overline{\boldsymbol{v}}(t), t)\right] \\
& +\sum_{i=1}^{N} \sum_{j=1}^{N} \tilde{\boldsymbol{x}}_{i}^{\mathrm{T}}(t) \boldsymbol{\Phi}_{i} \boldsymbol{R}_{i j} \boldsymbol{e}_{x j}(t)+\sum_{i=1}^{N} \sum_{j=1}^{N} \tilde{\boldsymbol{x}}_{i}^{\mathrm{T}}(t) \boldsymbol{\Phi}_{i} \boldsymbol{R}_{i j} \tilde{\boldsymbol{x}}_{j}(t) \\
& +\sum_{i=1}^{N} \sum_{j=1}^{N} \tilde{\boldsymbol{x}}_{i}^{\mathrm{T}}(t) \boldsymbol{\Phi}_{i} \boldsymbol{R}_{i j} \boldsymbol{e}_{v j}(t)+\sum_{i=1}^{N} \sum_{j=1}^{N} \tilde{\boldsymbol{x}}_{i}^{\mathrm{T}}(t) \boldsymbol{\Phi}_{i} \boldsymbol{R}_{i j} \tilde{\boldsymbol{v}}_{j}(t) \\
& +\sum_{i=1}^{N} \sum_{j=1}^{N} \tilde{\boldsymbol{v}}_{i}^{\mathrm{T}}(t) \boldsymbol{\Phi}_{i} \boldsymbol{R}_{i j} \boldsymbol{e}_{x j}(t)+\sum_{i=1}^{N} \sum_{j=1}^{N} \tilde{\boldsymbol{v}}_{i}^{\mathrm{T}}(t) \boldsymbol{\Phi}_{i} \boldsymbol{R}_{i j} \tilde{\boldsymbol{x}}_{j}(t) \\
& +\sum_{i=1}^{N} \sum_{j=1}^{N} \tilde{\boldsymbol{v}}_{i}^{\mathrm{T}}(t) \boldsymbol{\Phi}_{i} \boldsymbol{R}_{i j} \boldsymbol{e}_{v j}(t)+\sum_{i=1}^{N} \sum_{j=1}^{N} \tilde{\boldsymbol{v}}_{i}^{\mathrm{T}}(t) \boldsymbol{\Phi}_{i} \boldsymbol{R}_{i j} \tilde{\boldsymbol{v}}_{j}(t)
\end{aligned}
$$

Then, (12) can be rewritten as

$D^{\beta} V(t) \leq I_{1}+I_{2}+I_{3}+I_{4}$

where

$$
\begin{aligned}
I_{1}= & \alpha \sum_{i=1}^{N} \tilde{\boldsymbol{x}}_{i}^{\mathrm{T}}(t) \tilde{\boldsymbol{v}}_{i}(t)+\sum_{i=1}^{N} \sum_{j=1}^{N} \tilde{\boldsymbol{x}}_{i}^{\mathrm{T}}(t) \boldsymbol{\Phi}_{i} \boldsymbol{R}_{i j} \tilde{\boldsymbol{v}}_{j}(t) \\
& +\sum_{i=1}^{N} \sum_{j=1}^{N} \tilde{\boldsymbol{v}}_{i}^{\mathrm{T}}(t) \boldsymbol{\Phi}_{i} \boldsymbol{R}_{i j} \tilde{\boldsymbol{x}}_{j}(t) \\
I_{2}= & \sum_{i=1}^{N} \tilde{\boldsymbol{v}}_{i}^{\mathrm{T}}(t) \boldsymbol{\Phi}_{i} \tilde{\boldsymbol{v}}_{i}(t)+\sum_{i=1}^{N} \sum_{j=1}^{N} \tilde{\boldsymbol{x}}_{i}^{\mathrm{T}}(t) \boldsymbol{\Phi}_{i} \boldsymbol{R}_{i j} \tilde{\boldsymbol{x}}_{j}(t) \\
& +\sum_{i=1}^{N} \sum_{j=1}^{N} \tilde{\boldsymbol{v}}_{i}^{\mathrm{T}}(t) \boldsymbol{\Phi}_{i} \boldsymbol{R}_{i j} \tilde{\boldsymbol{v}}_{j}(t)
\end{aligned}
$$$$
I_{3}=\sum_{i=1}^{N} \sum_{j=1}^{N} \tilde{\boldsymbol{x}}_{i}^{\mathrm{T}}(t) \boldsymbol{\Phi}_{i} \boldsymbol{R}_{i j} \boldsymbol{e}_{x j}(t)+\sum_{i=1}^{N} \sum_{j=1}^{N} \tilde{\boldsymbol{x}}_{i}^{\mathrm{T}}(t) \boldsymbol{\Phi}_{i}
$$$$
\cdot \boldsymbol{R}_{i j} \boldsymbol{e}_{v j}(t)+\sum_{i=1}^{N} \sum_{j=1}^{N} \tilde{\boldsymbol{v}}_{i}^{\mathrm{T}}(t) \boldsymbol{\Phi}_{i} \boldsymbol{R}_{i j} \boldsymbol{e}_{x j}(t)
$$$$
+\sum_{i=1}^{N} \sum_{j=1}^{N} \tilde{\boldsymbol{v}}_{i}^{\mathrm{T}}(t) \boldsymbol{\Phi}_{i} \boldsymbol{R}_{i j} \boldsymbol{e}_{v j}(t)
$$

$$
\begin{aligned}
I_{4}= & \sum_{i=1}^{N} \tilde{\boldsymbol{x}}_{i}^{\mathrm{T}}(t) \boldsymbol{\Phi}_{i}\left[\boldsymbol{f}\left(\boldsymbol{x}_{i}(t), \boldsymbol{v}_{i}(t), t\right)-\boldsymbol{f}(\overline{\boldsymbol{x}}(t), \overline{\boldsymbol{v}}(t), t)\right] \\
& +\sum_{i=1}^{N} \tilde{\boldsymbol{v}}_{i}^{\mathrm{T}}(t) \boldsymbol{\Phi}_{i}\left[\boldsymbol{f}\left(\boldsymbol{x}_{i}(t), \boldsymbol{v}_{i}(t), t\right)-\boldsymbol{f}(\overline{\boldsymbol{x}}(t), \overline{\boldsymbol{v}}(t), t)\right]
\end{aligned}
$$

$$
\begin{aligned}
& I_{1}=\alpha \sum_{i=1}^{N} \tilde{\boldsymbol{x}}_{i}^{\mathrm{T}}(t) \tilde{\boldsymbol{v}}_{i}(t)+\sum_{i=1}^{N} \sum_{j=1}^{N} \sum_{k=1}^{n} \tilde{\boldsymbol{x}}_{i k}(t) \xi_{k i} r_{i j}^{k} \tilde{v}_{j k}(t) \\
& +\sum_{i=1}^{N} \sum_{j=1}^{N} \sum_{k=1}^{n} \tilde{v}_{i k}(t) \xi_{k i} r_{i j}^{k} \tilde{x}_{j k}(t) \\
& =\alpha \sum_{i=1}^{N} \tilde{\boldsymbol{x}}_{i}^{\mathrm{T}}(t) \tilde{\boldsymbol{v}}_{i}(t)+\frac{1}{2} \sum_{k=1}^{n} \tilde{\boldsymbol{\delta}} \boldsymbol{x}_{k}^{\mathrm{T}}(t)\left(\boldsymbol{\Xi}_{k} \boldsymbol{R}_{k}+\boldsymbol{R}_{k}^{\mathrm{T}} \boldsymbol{\Xi}_{k}\right) \\
& \cdot \tilde{\boldsymbol{v}}_{k}(t)+\frac{1}{2} \sum_{k=1}^{n} \tilde{\boldsymbol{\delta}}_{k}^{\mathrm{T}}(t)\left(\boldsymbol{\Xi}_{k} \boldsymbol{R}_{k}+\boldsymbol{R}_{k}^{\mathrm{T}} \boldsymbol{\Xi}_{k}\right) \tilde{\boldsymbol{\delta}}_{k}(t)
\end{aligned}
$$

where

$$
\tilde{\boldsymbol{x}}_{k}=\left[\tilde{x}_{1 k}, \tilde{x}_{2 k}, \ldots, \tilde{x}_{N k}\right]^{\mathrm{T}}, \tilde{\boldsymbol{\delta}}_{k}=\left[\tilde{v}_{1 k}, \tilde{v}_{2 k}, \ldots, \tilde{v}_{N k}\right]^{\mathrm{T}} \text {. }
$$

According to the definition of $\boldsymbol{Q}_{k}$, we conclude that the columns of $\boldsymbol{Q}_{k}$ form a basis of the subspace $\boldsymbol{E}_{k}$, where $\boldsymbol{E}_{k}=\left\{\boldsymbol{z}=\left[z_{1}, z_{2}, \ldots, z_{N}\right]^{\mathrm{T}} \in \mathbf{R}^{N}: \sum_{i=1}^{N} \xi_{k i} z_{i}=0\right\}$. Therefore, we obtain $\tilde{\boldsymbol{\delta}}_{k}, \tilde{\boldsymbol{\delta}}_{k} \in \boldsymbol{E}_{k}$. Hence, there exists $\hat{\boldsymbol{\delta}} \hat{\boldsymbol{v}}_{k}, \boldsymbol{\delta} \hat{\boldsymbol{x}}_{k} \in \mathbf{R}^{N-1}$, such that $\tilde{\boldsymbol{\delta}}_{k}=\boldsymbol{Q}_{k} \boldsymbol{\delta} \hat{\boldsymbol{v}}_{k}$ and $\tilde{\boldsymbol{x}}_{k}=\boldsymbol{Q}_{k} \boldsymbol{\delta} \hat{\boldsymbol{x}}_{k}$. Thus, (13) follows that 


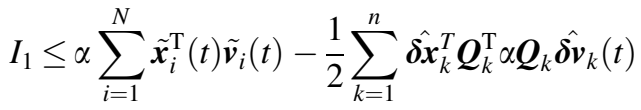

$$
\begin{aligned}
& -\frac{1}{2} \sum_{k=1}^{n} \hat{\boldsymbol{\delta}} \hat{\boldsymbol{v}}_{k}^{\mathrm{T}} \boldsymbol{Q}_{k}^{\mathrm{T}} \alpha \boldsymbol{Q}_{k} \hat{\boldsymbol{\delta}} \boldsymbol{x}_{k}(t) \\
& =\alpha \sum_{i=1}^{N} \tilde{\boldsymbol{x}}_{i}^{\mathrm{T}}(t) \tilde{\boldsymbol{v}}_{i}(t)-\frac{\alpha}{2} \sum_{k=1}^{n} \tilde{\boldsymbol{\delta}}_{k}^{\mathrm{T}}(t) \tilde{\boldsymbol{\delta}}_{k}(t) \\
& -\frac{\alpha}{2} \sum_{k=1}^{n} \tilde{\boldsymbol{\delta}}_{k}^{\mathrm{T}}(t) \tilde{\boldsymbol{\delta}}_{k}(t) \\
& =\alpha \sum_{i=1}^{N} \tilde{\boldsymbol{x}}_{i}^{\mathrm{T}}(t) \tilde{\boldsymbol{v}}_{i}(t)-\frac{\alpha}{2} \sum_{i=1}^{N} \tilde{\boldsymbol{x}}_{i}^{\mathrm{T}}(t) \tilde{\boldsymbol{v}}_{i}(t) \\
& -\frac{\alpha}{2} \sum_{i=1}^{N} \tilde{\boldsymbol{v}}_{i}^{\mathrm{T}}(t) \tilde{\boldsymbol{x}}_{i}(t) \leq 0 \\
& I_{2}=\sum_{i=1}^{N} \tilde{\boldsymbol{v}}_{i}^{\mathrm{T}}(t) \boldsymbol{\Phi}_{i} \tilde{\boldsymbol{v}}_{i}(t)+\sum_{i=1}^{N} \sum_{j=1}^{N} \sum_{k=1}^{n} \tilde{x}_{i k}(t) \xi_{k i} r_{i j}^{k} \tilde{j}_{j k}(t) \\
& +\sum_{i=1}^{N} \sum_{j=1}^{N} \sum_{k=1}^{n} \tilde{v}_{i k}(t) \xi_{k i} r_{i j}^{k} \tilde{v}_{j k}(t) \\
& \leq \sum_{i=1}^{N} \tilde{\boldsymbol{v}}_{i}^{\mathrm{T}}(t) \boldsymbol{\Phi}_{i} \tilde{\boldsymbol{v}}_{i}(t)-\frac{\alpha}{2} \sum_{i=1}^{N} \tilde{\boldsymbol{x}}_{i}^{\mathrm{T}}(t) \tilde{\boldsymbol{x}}_{i}(t) \\
& -\frac{\alpha}{2} \sum_{i=1}^{N} \tilde{\boldsymbol{v}}_{i}^{\mathrm{T}}(t) \tilde{\boldsymbol{v}}_{i}(t) \\
& =-\frac{\alpha}{2} \sum_{i=1}^{N} \tilde{\boldsymbol{x}}_{i}^{\mathrm{T}}(t) \tilde{\boldsymbol{x}}_{i}(t)+\sum_{i=1}^{N} \tilde{\boldsymbol{v}}_{i}^{\mathrm{T}}(t)\left(\boldsymbol{\Phi}_{i}-\frac{\alpha}{2} \boldsymbol{I}_{n}\right) \tilde{\boldsymbol{v}}_{i}(t)
\end{aligned}
$$

From the definitions of measurement errors, we found that $\boldsymbol{e}_{x i}(t)$ and $\boldsymbol{e}_{v i}(t)$ will be set to zeros at each event sampling instant $t_{k}^{i}$. The next sampling time instant $t_{k+1}^{i}$ is the moment when the term $\left\|\boldsymbol{e}_{x i}(t)\right\|_{1}+\left\|\boldsymbol{e}_{v i}(t)\right\|_{1}$ becomes the time-varying threshold

$$
\begin{aligned}
& \varphi_{1} \sum_{j \in N_{i}}\left[\left\|\boldsymbol{R}_{i j}\left(\boldsymbol{x}_{i}\left(t_{k}^{i}\right)-\boldsymbol{x}_{j}\left(t_{k^{\prime}(t)}^{j}\right)\right)\right\|_{1}\right. \\
& \left.\quad+\left\|\boldsymbol{R}_{i j}\left(\boldsymbol{v}_{i}\left(t_{k}^{i}\right)-\boldsymbol{v}_{j}\left(t_{k^{\prime}(t)}^{j}\right)\right)\right\|_{1}\right]+\varphi_{2} e^{-\gamma t}
\end{aligned}
$$

Then, for all $t \geq 0$,

$$
\begin{aligned}
& \left\|\boldsymbol{e}_{x i}(t)\right\|_{1}+\left\|\boldsymbol{e}_{v i}(t)\right\|_{1} \\
& \leq \varphi_{1} \sum_{j \in N_{i}}\left[\left\|\boldsymbol{R}_{i j}\left(\boldsymbol{x}_{i}\left(t_{k}^{i}\right)-\boldsymbol{x}_{j}\left(t_{k^{\prime}(t)}^{j}\right)\right)\right\|_{1}\right] \\
& \quad+\varphi_{1} \sum_{j \in N_{i}}\left[\left\|\boldsymbol{R}_{i j}\left(\boldsymbol{v}_{i}\left(t_{k}^{i}\right)-\boldsymbol{v}_{j}\left(t_{k^{\prime}(t)}^{j}\right)\right)\right\|_{1}\right]+\varphi_{2} e^{-\gamma t} \\
& \leq \varphi_{1} \sum_{j \in N_{i}}\left[\left\|\boldsymbol{R}_{i j}\right\|_{1}\left\|\left(\boldsymbol{x}_{i}\left(t_{k}^{i}\right)-\boldsymbol{x}_{j}\left(t_{k^{\prime}(t)}^{j}\right)\right)\right\|_{1}\right] \\
& \quad+\varphi_{1} \sum_{j \in N_{i}}\left[\left\|\boldsymbol{R}_{i j}\right\|_{1}\left\|\left(\boldsymbol{v}_{i}\left(t_{k}^{i}\right)-\boldsymbol{v}_{j}\left(t_{k^{\prime}(t)}^{j}\right)\right)\right\|_{1}\right]+\varphi_{2} e^{-\gamma t} \\
& \leq \varphi_{1} N_{M} \bar{r}\left(\left\|\boldsymbol{e}_{x}(t)\right\|_{1}+\left\|\boldsymbol{e}_{v}(t)\right\|_{1}\right)+\varphi_{2} e^{-\gamma t} \\
& \quad+\varphi_{1} N_{M} \bar{r}\left(\|\tilde{\boldsymbol{x}}(t)\|_{1}+\|\tilde{\boldsymbol{v}}(t)\|_{1}\right)
\end{aligned}
$$

where $\bar{r}=\max _{1 \leq i \leq N, j \in N_{i}, 1 \leq k \leq n} r_{i j}^{k}, N_{M}=\max _{1 \leq i \leq N}\left|N_{i}\right|$. By adding $i$ from 1 to $N$, we can obtain from (16) that

$$
\begin{aligned}
& \left\|\boldsymbol{e}_{x}(t)\right\|_{1}+\left\|\boldsymbol{e}_{v}(t)\right\|_{1} \leq \varphi_{1} N_{M} N \bar{r}\left(\left\|\boldsymbol{e}_{x}(t)\right\|_{1}+\left\|\boldsymbol{e}_{v}(t)\right\|_{1}\right) \\
& \quad+\varphi_{1} N_{M} N \bar{r}\left(\|\tilde{\boldsymbol{x}}(t)\|_{1}+\|\tilde{\boldsymbol{v}}(t)\|_{1}\right)+\varphi_{2} N e^{-\gamma t}
\end{aligned}
$$

i.e.,

$$
\begin{aligned}
\| \boldsymbol{e}_{x}(t) & \left\|_{1}+\right\| \boldsymbol{e}_{v}(t) \|_{1} \\
\leq & \frac{\varphi_{1} N_{M} N \bar{r}}{1-\varphi_{1} N_{M} N \bar{r}}\left(\|\tilde{\boldsymbol{x}}(t)\|_{1}+\|\tilde{\boldsymbol{v}}(t)\|_{1}\right) \\
& \quad+\frac{\varphi_{2} N}{1-\varphi_{1} N_{M} N \bar{r}} e^{-\gamma t} \\
= & \phi_{1}\left(\|\tilde{\boldsymbol{x}}(t)\|_{1}+\|\tilde{\boldsymbol{v}}(t)\|_{1}\right)+\phi_{2} e^{-\gamma t}
\end{aligned}
$$

Thus, we can obtain from $I_{3}$ that

$$
\begin{aligned}
I_{3}= & \sum_{i=1}^{N} \sum_{j=1}^{N} \tilde{\boldsymbol{x}}_{i}^{\mathrm{T}}(t) \boldsymbol{\Pi}_{i j}\left(\boldsymbol{e}_{x j}(t)+\boldsymbol{e}_{v j}(t)\right) \\
& +\sum_{i=1}^{N} \sum_{j=1}^{N} \tilde{\boldsymbol{v}}_{i}^{\mathrm{T}}(t) \boldsymbol{\Pi}_{i j}\left(\boldsymbol{e}_{x j}(t)+\boldsymbol{e}_{v j}(t)\right) \\
\leq & \left|\sum_{i=1}^{N} \sum_{j=1}^{N} \tilde{\boldsymbol{x}}_{i}^{\mathrm{T}}(t) \boldsymbol{\Pi}_{i j}\left(\boldsymbol{e}_{x j}(t)+\boldsymbol{e}_{v j}(t)\right)\right| \\
& +\left|\sum_{i=1}^{N} \sum_{j=1}^{N} \tilde{\boldsymbol{v}}_{i}^{\mathrm{T}}(t) \boldsymbol{\Pi}_{i j}\left(\boldsymbol{e}_{x j}(t)+\boldsymbol{e}_{v j}(t)\right)\right| \\
\leq & M|| \tilde{\boldsymbol{x}}^{\mathrm{T}}(t) \|_{1}\left(\left\|\boldsymbol{e}_{x}(t)\right\|_{1}+\left\|\boldsymbol{e}_{v}(t)\right\|_{1}\right) \\
& +M\left\|\tilde{\boldsymbol{v}}^{\mathrm{T}}(t)\right\|_{1}\left(\left\|\boldsymbol{e}_{x}(t)\right\|_{1}+\left\|\boldsymbol{e}_{v}(t)\right\|_{1}\right) \\
= & {\left[2 M \phi_{1} N n+\frac{M^{2} \phi_{2}^{2} N n}{4 \kappa^{2}}\right] \tilde{\boldsymbol{x}}^{\mathrm{T}}(t) \tilde{\boldsymbol{x}}(t)+\eta e^{-2 \gamma t} } \\
& +\left[2 M \phi_{1} N n+\frac{M^{2} \phi_{2}^{2} N n}{4 \sigma^{2}}\right] \tilde{\boldsymbol{v}}^{\mathrm{T}}(t) \tilde{\boldsymbol{v}}(t)
\end{aligned}
$$


where

$$
\begin{aligned}
\boldsymbol{\Pi}_{i j}= & \boldsymbol{\Phi}_{i} \boldsymbol{R}_{i j} ; \kappa>0 ; \sigma>0 ; \eta=\kappa^{2}+\sigma^{2} . \\
I_{4}= & \sum_{i=1}^{N} \tilde{\boldsymbol{x}}_{i}^{\mathrm{T}}(t) \boldsymbol{\Phi}_{i}\left[\boldsymbol{f}\left(\boldsymbol{x}_{i}(t), \boldsymbol{v}_{i}(t), t\right)-\boldsymbol{f}(\overline{\boldsymbol{x}}(t), \overline{\boldsymbol{v}}(t), t)\right] \\
& +\sum_{i=1}^{N} \tilde{\boldsymbol{v}}_{i}^{\mathrm{T}}(t) \boldsymbol{\Phi}_{i}\left[\boldsymbol{f}\left(\boldsymbol{x}_{i}(t), \boldsymbol{v}_{i}(t), t\right)-\boldsymbol{f}(\overline{\boldsymbol{x}}(t), \overline{\boldsymbol{v}}(t), t)\right] \\
\leq & \frac{1}{2} \sum_{i=1}^{N} \tilde{\boldsymbol{x}}_{i}^{\mathrm{T}}(t) \boldsymbol{\Phi}_{i} \tilde{\boldsymbol{x}}_{i}(t)+\frac{1}{2} \sum_{i=1}^{N} \tilde{\boldsymbol{v}}_{i}^{\mathrm{T}}(t) \boldsymbol{\Phi}_{i} \tilde{\boldsymbol{v}}_{i}(t) \\
& +\sum_{i=1}^{N}\left[\boldsymbol{f}\left(\boldsymbol{x}_{i}(t), \boldsymbol{v}_{i}(t), t\right)-\boldsymbol{f}(\overline{\boldsymbol{x}}(t), \overline{\boldsymbol{v}}(t), t)\right]^{\mathrm{T}} \boldsymbol{\Phi}_{i} \\
& {\left[\boldsymbol{f}\left(\boldsymbol{x}_{i}(t), \boldsymbol{v}_{i}(t), t\right)-\boldsymbol{f}(\overline{\boldsymbol{x}}(t), \overline{\boldsymbol{v}}(t), t)\right] } \\
\leq & \left(l_{1}^{2}+l_{1} l_{2}+\frac{1}{2}\right) \sum_{i=1}^{N} \tilde{\boldsymbol{x}}_{i}^{\mathrm{T}}(t) \boldsymbol{\Phi}_{i} \tilde{\boldsymbol{x}}_{i}(t) \\
& +\left(l_{2}^{2}+l_{1} l_{2}+\frac{1}{2}\right) \sum_{i=1}^{N} \tilde{\boldsymbol{v}}_{i}^{\mathrm{T}}(t) \boldsymbol{\Phi}_{i} \tilde{\boldsymbol{v}}_{i}(t)
\end{aligned}
$$

From (14),(15),(17) and (18), we can obtain the following:

$$
\begin{aligned}
& D^{\beta} V(t) \leq \sum_{i=1}^{N} \tilde{\boldsymbol{x}}_{i}^{\mathrm{T}}(t)\left[\left(-\frac{\alpha}{2}+2 M \phi_{1} N n+\frac{M^{2} \phi_{2}^{2} N n}{4 \kappa^{2}}\right)\right. \\
&\left.\cdot \tilde{\boldsymbol{x}}_{i}(t)\right]+\sum_{i=1}^{N} \tilde{\boldsymbol{x}}_{i}^{\mathrm{T}}(t)\left[\left(l_{1}^{2}+l_{1} l_{2}+\frac{1}{2}\right) \boldsymbol{\Phi}_{i}\right] \tilde{\boldsymbol{x}}_{i}(t) \\
&+ \sum_{i=1}^{N} \tilde{\boldsymbol{v}}_{i}^{\mathrm{T}}(t)\left[-\frac{\alpha}{2}+2 M \phi_{1} N n+\frac{M^{2} \phi_{2}^{2} N n}{4 \sigma^{2}}\right] \tilde{\boldsymbol{v}}_{i}(t) \\
&+ \sum_{i=1}^{N} \tilde{\boldsymbol{x}}_{i}^{\mathrm{T}}(t)\left[\left(l_{2}^{2}+l_{1} l_{2}+\frac{3}{2}\right) \boldsymbol{\Phi}_{i}\right] \tilde{\boldsymbol{v}}_{i}(t) \\
&+\left(\kappa^{2}+\sigma^{2}\right) e^{-2 \gamma t} \\
&=-\sum_{i=1}^{N}\left(\tilde{\boldsymbol{x}}_{i}^{\mathrm{T}}(t), \tilde{\boldsymbol{v}}_{i}^{\mathrm{T}}(t)\right) \boldsymbol{\Theta}\left[\begin{array}{c}
\tilde{\boldsymbol{x}}_{i}(t) \\
\tilde{\boldsymbol{v}}_{i}(t)
\end{array}\right]+\eta e^{-2 \gamma t}
\end{aligned}
$$

where

$$
\boldsymbol{\Theta}=\left[\begin{array}{cc}
\mu_{1} \boldsymbol{I}_{n}-\left(l_{1}^{2}+l_{1} l_{2}+\frac{1}{2}\right) \boldsymbol{\Phi}_{i} & \mathbf{0} \\
\mathbf{0} & \mu_{2} I_{n}-\left(l_{2}^{2}+l_{1} l_{2}+\frac{3}{2}\right) \boldsymbol{\Phi}_{i}
\end{array}\right] .
$$

From the condition of the theorem, we know that $\boldsymbol{\Theta}>\mathbf{0}$. Choosing $0<\theta<\frac{\lambda_{\min }(\boldsymbol{\Theta})}{\lambda_{\max }(\boldsymbol{P})}$, we have

$D^{\beta} V(t) \leq-\theta V(t)+h(t)$

where $h(t)=\eta e^{-2 \gamma t} ; \boldsymbol{P}=\left[\begin{array}{cc}\alpha \boldsymbol{I}_{n} & \boldsymbol{\Phi}_{i} \\ \boldsymbol{\Phi}_{i} & \boldsymbol{\Phi}_{i}\end{array}\right]$. Therefore, there exists a non-negative function $q(t)$ such that $D^{\beta} V(t)+$ $q(t)=-\theta V(t)+h(t)$. We can obtain its solution by the Laplace transform method [44]. Then,

$$
\begin{aligned}
V(t)= & E_{\beta}\left(-\theta t^{\beta}\right) V(0)+h(t) * t^{\beta-1} E_{\beta, \beta}\left(-\theta t^{\beta}\right) \\
& -q(t) * t^{\beta-1} E_{\beta, \beta}\left(-\theta t^{\beta}\right) \\
= & E_{\beta}\left(-\theta t^{\beta}\right) V(0)-q(t) * t^{\beta-1} E_{\beta, \beta}\left(-\theta t^{\beta}\right) \\
& \quad+\int_{0}^{t}(t-\tau)^{\beta-1} E_{\beta, \beta}\left(-\theta(t-\tau)^{\beta}\right) h(\tau) \mathrm{d} \tau
\end{aligned}
$$

where $*$ stands for the convolution between two functions,

$$
\begin{aligned}
& E_{\beta}\left(-\theta t^{\beta}\right)=\sum_{k=0}^{\infty} \frac{\left(-\theta t^{\beta}\right)^{k}}{\Gamma(k \beta+1)}, \\
& E_{\beta, \beta}\left(-\theta(t-\tau)^{\beta}\right)=\sum_{k=0}^{\infty} \frac{\left(-\theta(t-\tau)^{\beta}\right)^{k}}{\Gamma(k \beta+\beta)} .
\end{aligned}
$$

Because the non-negative function $q(t) \geq 0, t^{\beta-1} \geq 0$ and $E_{\beta, \beta}\left(-\theta t^{\beta}\right) \geq 0$, we have $q(t) * t^{\beta-1} E_{\beta, \beta}\left(-\theta t^{\beta}\right) \geq 0$. Let $\tau=$ $t, \tau_{0}=\theta^{\beta}$, therefore, $\tau / \tau_{0} \rightarrow \infty$ when $\tau \rightarrow \infty$. Then, $E_{\beta}\left[-\left(\tau / \tau_{0}\right)^{\beta}\right] \sim\left(\tau / \tau_{0}\right)^{-\beta} / \Gamma(1-\beta)$ from Lemma 5. Thus, $\lim _{t \rightarrow \infty} E_{\beta}\left(-\theta t^{\beta}\right) V(0)=0$. It is noteworthy that $h(t)=\eta e^{-2 \gamma t}$.

Therefore, we have

$$
\begin{aligned}
V(t)= & E_{\beta}\left(-\theta t^{\beta}\right) V(0) \\
& +\varrho(t)-q(t) * t^{\beta-1} E_{\beta, \beta}\left(-\theta t^{\beta}\right)
\end{aligned}
$$

where

$$
\varrho(t)=\eta \int_{0}^{t}(t-\tau)^{\beta-1} E_{\beta, \beta}\left(-\theta(t-\tau)^{\beta}\right) e^{-2 \gamma \tau} \mathrm{d} \tau .
$$

With variable substitution,

$$
\varrho(t)=\eta e^{-2 \gamma t} \int_{0}^{t} \tau^{\beta-1} E_{\beta, \beta}\left(-\theta \tau^{\beta}\right) e^{2 \gamma \tau} \mathrm{d} \tau .
$$

We then denote $\varpi(t)=\tau^{\beta-1} E_{\beta, \beta}\left(-\theta \tau^{\beta}\right)$. It is obvious that $\lim _{t \rightarrow \infty} \varpi(t)=0$. Similar to the analysis in [47], one can obtain that, $\lim _{t \rightarrow \infty} \varrho(t)=0$. Subsequently, $\lim _{t \rightarrow \infty} V(t)=0$. Thus, the consensus in the fractional-order MAS (6) is achieved asymptotically, implying that the position and speed of every agent will approach a time-varying common state.

Next, we will show that the Zeno behavior would be excluded. Therefore, there exists a strictly positive number such that for each $i, i=1,2, \ldots, N$, we have $t_{k+1}^{i}-t_{k}^{i}>\tau$ for all $k \in N$. For $t \in\left[t_{k}^{i}, t_{k+1}^{i}\right)$, letting $\boldsymbol{u}_{i}\left(t_{k}^{i}, t_{k^{\prime}(t)}^{j}\right)=$ $\sum_{j=1, j \neq i}^{N}\left[\boldsymbol{R}_{i j}\left(\boldsymbol{x}_{j}\left(t_{k^{\prime}}^{j}\right)-\boldsymbol{x}_{i}\left(t_{k}^{i}\right)\right)+\boldsymbol{R}_{i j}\left(\boldsymbol{v}_{j}\left(t_{k^{\prime}}^{j}\right)-\boldsymbol{v}_{i}\left(t_{k}^{i}\right)\right)\right]$, 


$$
\begin{aligned}
D_{+}^{\alpha} & \left(\left\|\boldsymbol{e}_{x i}(t)\right\|_{1}+\left\|\boldsymbol{e}_{v i}(t)\right\|_{1}\right) \leq\left\|\dot{\boldsymbol{e}}_{x i}(t)\right\|_{1}+\left\|\dot{\boldsymbol{e}}_{v i}(t)\right\|_{1} \\
= & \left\|\boldsymbol{v}_{i}(t)\right\|_{1}+\left\|f\left(\boldsymbol{x}_{i}(t), \boldsymbol{v}_{i}(t), t\right)+\boldsymbol{u}_{i}\left(t_{k}^{i}, t_{k^{\prime}(t)}^{j}\right)\right\|_{1} \\
\leq & \left\|\boldsymbol{e}_{v i}(t)+\boldsymbol{v}_{i}\left(t_{k}^{i}\right)\right\|_{1}+l_{1}\left\|\boldsymbol{x}_{i}\left(t_{k}^{i}\right)+\boldsymbol{e}_{x i}(t)\right\|_{1}+l_{2} \| \boldsymbol{e}_{v i}(t) \\
& +\boldsymbol{v}_{i}\left(t_{k}^{i}\right)\left\|_{1}+\right\| \boldsymbol{u}_{i}\left(t_{k}^{i}, t_{k^{\prime}(t)}^{j}\right) \|_{1} \\
\leq & \max \left\{l_{1}, 1+l_{2}\right\}\left[\left\|\boldsymbol{e}_{x i}(t)\right\|_{1}+\left\|\boldsymbol{e}_{v i}(t)\right\|_{1}\right]+l_{1}\left\|\boldsymbol{x}_{i}\left(t_{k}^{i}\right)\right\|_{1} \\
& +\left(1+l_{2}\right)\left\|\boldsymbol{v}_{i}\left(t_{k}^{i}\right)\right\|_{1}+\left\|\boldsymbol{u}_{i}\left(t_{k}^{i}, t_{k^{\prime}(t)}^{j}\right)\right\|_{1} \\
= & l\left[\left\|\boldsymbol{e}_{x i}(t)\right\|_{1}+\left\|\boldsymbol{e}_{v i}(t)\right\|_{1}\right]+l_{1}\left\|\boldsymbol{x}_{i}\left(t_{k}^{i}\right)\right\|_{1} \\
& +\left(1+l_{2}\right)\left\|\boldsymbol{v}_{i}\left(t_{k}^{i}\right)\right\|_{1}+\left\|\boldsymbol{u}_{i}\left(t_{k}^{i}, t_{k^{\prime}(t)}^{j}\right)\right\|_{1} .
\end{aligned}
$$

Let $\chi\left(t_{k}^{i}, t_{k^{\prime}(t)}^{j}\right)=l_{1}\left\|\boldsymbol{x}_{i}\left(t_{k}^{i}\right)\right\|_{1}+\left(1+l_{2}\right)\left\|\boldsymbol{v}_{i}\left(t_{k}^{i}\right)\right\|_{1}+\| \boldsymbol{u}_{i}\left(t_{k}^{i}\right.$, $\left.t_{k^{\prime}(t)}^{j}\right) \|_{1}$, and integrating from $t_{k}^{i}$ to $t$, we obtain $t \in\left[t_{k}^{i}, t_{k+1}^{i}\right)$,

$$
\left\|\boldsymbol{e}_{x i}(t)\right\|_{1}+\left\|\boldsymbol{e}_{v i}(t)\right\|_{1} \leq \frac{\chi\left(t_{k}^{i}, t_{k^{\prime}(t)}^{j}\right)}{l} e^{l\left(t-t_{k}^{i}\right)}-\frac{\chi\left(t_{k}^{i}, t_{k^{\prime}(t)}^{j}\right)}{l},
$$

Using $t=t_{k+1}^{i-}$, we have

$$
\begin{aligned}
& \left\|\boldsymbol{e}_{x i}\left(t_{k+1}^{i-}\right)\right\|_{1}+\left\|\boldsymbol{e}_{v i}\left(t_{k+1}^{i-}\right)\right\|_{1} \\
& \quad \leq \frac{\chi\left(t_{k}^{i}, t_{k^{\prime}(t)}^{j}\right)}{l} e^{l\left(t_{k+1}^{i}-t_{k}^{i}\right)}-\frac{\chi\left(t_{k}^{i}, t_{k^{\prime}(t)}^{j}\right)}{l} .
\end{aligned}
$$

It is noteworthy that the next sampling time instant $t_{k+1}^{i}$ will yield the following equality:

$$
\begin{aligned}
& \left\|\boldsymbol{e}_{x i}\left(t_{k+1}^{i-}\right)\right\|_{1}+\left\|\boldsymbol{e}_{v i}\left(t_{k+1}^{i-}\right)\right\|_{1} \\
& >\varphi_{1} \sum_{j \in N_{i}}\left[\left\|\boldsymbol{x}_{i}\left(t_{k}^{i}-\boldsymbol{x}_{j}\left(t_{k^{\prime}(t)}^{j}\right)\right)\right\|+\left\|\boldsymbol{v}_{i}\left(t_{k}^{i}-\boldsymbol{v}_{j}\left(t_{k^{\prime}(t)}^{j}\right)\right)\right\|\right] \\
& \quad+\varphi_{2} e^{-\gamma \gamma_{k+1}^{i}}=\varphi_{1}\left\|\boldsymbol{u}_{i}\left(t_{k}^{i}, t_{k^{\prime}(t)}^{j}\right)\right\|_{1}+\varphi_{2} e^{-\gamma t_{k+1}^{i}}
\end{aligned}
$$

Thus, we have

$$
\begin{aligned}
& \varphi_{1}\left\|\boldsymbol{u}_{i}\left(t_{k}^{i}, t_{k^{\prime}(t)}^{j}\right)\right\|_{1}+\varphi_{2} e^{-\gamma t_{k+1}^{i}} \\
& <\frac{\chi\left(t_{k}^{i}, t_{k^{\prime}(t)}^{j}\right)}{l} e^{l\left(t_{k+1}^{i}-t_{k}^{i}\right)}-\frac{\chi\left(t_{k}^{i}, t_{k^{\prime}(t)}^{j}\right)}{l}
\end{aligned}
$$

i.e.,

$$
\begin{array}{r}
\varphi_{1}\left\|\boldsymbol{u}_{i}\left(t_{k}^{i}, t_{k^{\prime}(t)}^{j}\right)\right\|_{1}+\varphi_{2} e^{-\gamma t_{k}^{i}} e^{-\gamma \tau} \\
<\frac{\chi\left(t_{k}^{i}, t_{k^{\prime}(t)}^{j}\right)}{l} e^{l \tau}-\frac{\chi\left(t_{k}^{i}, t_{k^{\prime}(t)}^{j}\right)}{l}
\end{array}
$$

where $\tau=t_{k+1}^{i}-t_{k}^{i}$. Let

$$
\begin{aligned}
& k_{1}=\varphi_{1}\left\|\boldsymbol{u}_{i}\left(t_{k}^{i}, t_{k^{\prime}(t)}^{j}\right)\right\|_{1}+\frac{\chi\left(t_{k}^{i}, t_{k^{\prime}(t)}^{j}\right)}{l} \\
& k_{2}=\varphi_{2} e^{-\gamma t_{k}^{i}}, \quad k_{3}=\frac{\chi\left(t_{k}^{i}, t_{k^{\prime}(t)}^{j}\right)}{l}
\end{aligned}
$$

Then, we have

$$
k_{1}+k_{2} e^{-\gamma \tau}<k_{3} e^{l \tau}
$$

Because $k_{1}+k_{2}>k_{3}$, a strictly positive $\tau$ must exist that satisfies the equality $k_{1}+k_{2} e^{-\gamma t}=k_{3} e^{l t}$, as illustrated in Fig. 1. The Zeno behavior is excluded for all agents during the sampling process, i.e., $t_{k+1}^{i}-t_{k}^{i}>\tau$ for all $k \in N$ and $i=1,2, \ldots, N$. The proof is completed.

When the information among the agents can be perfectly transmitted through the communication system, meaning the channel matrix is equal to an identity matrix $\boldsymbol{I}_{n}$, from the definitions of $\boldsymbol{R}_{i j}, \boldsymbol{R}_{k}, \boldsymbol{\Phi}_{i}$ in the former part, they can be redefined as $\quad \boldsymbol{R}_{i j}=a_{i j} \boldsymbol{I}_{n}, \boldsymbol{R}_{k}=-\boldsymbol{L}, \boldsymbol{\Phi}_{i}=\xi_{i} \boldsymbol{I}_{n}$, and $\boldsymbol{\Xi}_{k}=$ $\boldsymbol{\Xi}=\operatorname{diag}\left\{\xi_{1}, \xi_{2}, \ldots, \xi_{N}\right\}$, respectively, where $\boldsymbol{L}$ is the Laplacian matrix of the network. Subsequently, the fractional-order MAS by the event-trigger sampling scheme would be

$$
\left\{\begin{array}{l}
D^{\beta} \boldsymbol{x}_{i}(t)=\boldsymbol{v}_{i}(t) \\
D^{\beta} \boldsymbol{v}_{i}(t)=\boldsymbol{u}_{i}(t) \\
\boldsymbol{u}_{i}(t)=\boldsymbol{f}\left(\boldsymbol{x}_{i}(t), \boldsymbol{v}_{i}(t), t\right) \\
\quad-\sum_{j=1, j \neq i}^{N}\left[a_{i j}\left(\boldsymbol{x}_{i}\left(t_{k}^{i}\right)-\boldsymbol{x}_{j}\left(t_{k^{\prime}}^{j}\right)\right)+a_{i j}\left(\boldsymbol{v}_{i}\left(t_{k}^{i}\right)-\boldsymbol{v}_{j}\left(t_{k^{\prime}}^{j}\right)\right)\right]
\end{array}\right.
$$

Corollary 1 Suppose Assumption 1 holds and $t_{0}^{i}=t_{0}=$ 0 under the event condition. $\boldsymbol{R}_{k}$ is irreducible. Therefore, the consensus in network (21) is achieved under the designed control input if there exists a scalar $\alpha>0$ satisfying the following LMIs:

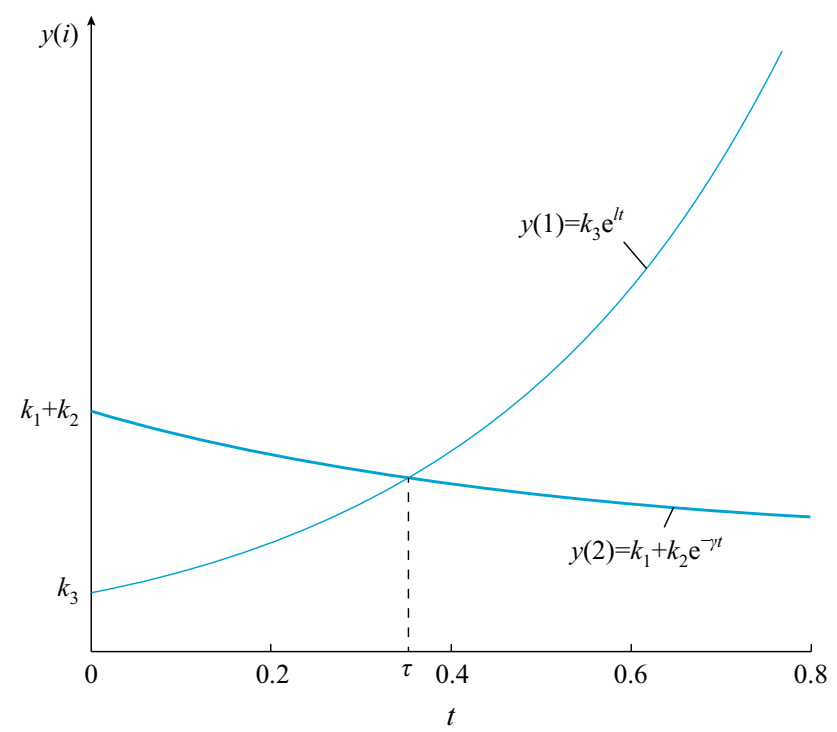

Fig. 1 Solution of the equality for $\tau$ 


$$
\left\{\begin{array}{l}
\boldsymbol{Q}^{\mathrm{T}}\left(\boldsymbol{\Xi} \boldsymbol{L}+\boldsymbol{L}^{\mathrm{T}} \boldsymbol{\Xi}-\alpha \boldsymbol{I}_{N}\right) \boldsymbol{Q}>\mathbf{0} \\
\mu_{1}>0 \\
\mu_{2}>0 \\
\alpha-\xi_{i}>0
\end{array}\right.
$$

where

$$
\begin{aligned}
& \mu_{1}=\frac{\alpha}{2}-2 M \phi_{1} N n-\frac{M^{2} \phi_{2}^{2} N n}{4 \kappa^{2}}-\left(l_{1}^{2}+l_{1} l_{2}+\frac{1}{2}\right) \xi_{i}, \\
& \mu_{2}=\frac{\alpha}{2}-2 M \phi_{1} N n-\frac{M^{2} \phi_{2}^{2} N n}{4 \sigma^{2}}-\left(l_{2}^{2}+l_{1} l_{2}+\frac{3}{2}\right) \xi_{i}, \\
& \bar{r}=\max _{1 \leq i \leq N, j \in N_{i}} a_{i j}, M=\max _{1 \leq i, j \leq N} \xi_{i} a_{i j}, \gamma>0, \\
& \phi_{1}=\frac{\varphi_{1} N_{M} N \bar{r}}{1-\varphi_{1} N_{M} N \bar{r}}, \phi_{2}=\frac{\varphi_{2} N}{1-\varphi_{1} N_{M} N \bar{r}} .
\end{aligned}
$$

\section{Simulation example}

In this section, a numerical example is presented to demonstrate the effectiveness of the primary results.

Consider a 5-generator 14-bus cyber-physical power system, as shown in Fig. 2. The related data are obtained from IEEE 5-generator 14-bus standard test system. Each of the generators is assigned with an agent such that it can obtain the others information through the communication of the agents, which is shown in Fig. 3. Here, the double arrow solid line indicates the cyber network, and the single arrow solid line represents the physical network. Based on this distributed multi-agent mechanism, the power cyber networks and physical networks are effectively integrated together. Supposing that each agent (generator) contains two levels of information, i.e., $N=5, n=2$. The

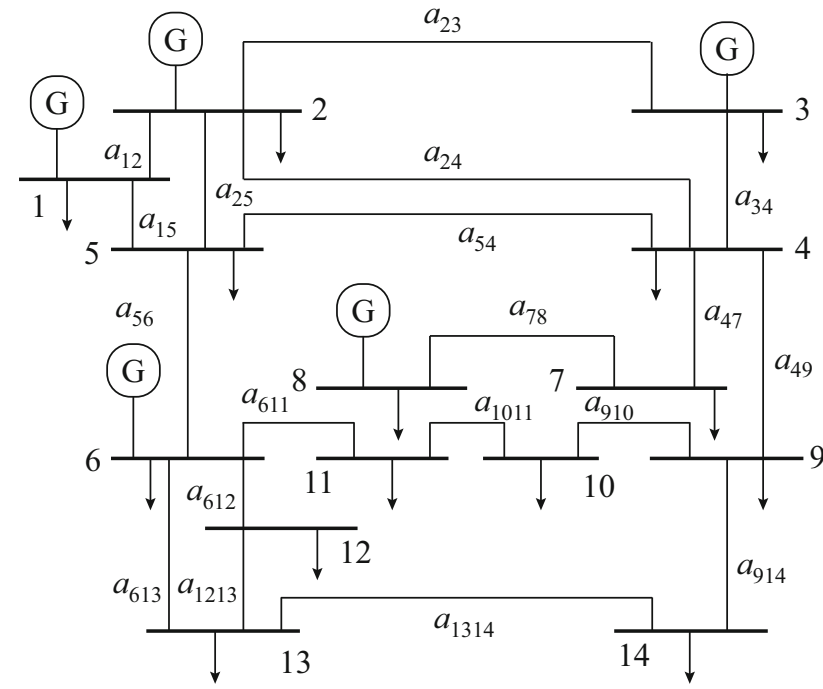

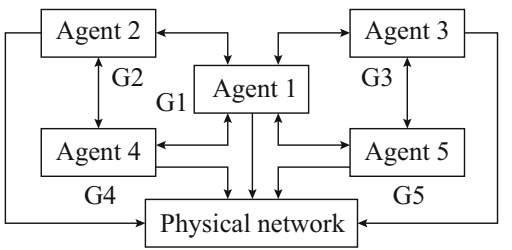

Fig. 3 Physical interconnection and cyber interconnection of CPPS

connection of each agent can be represented by $\boldsymbol{A}=\left[\begin{array}{lllll}0 & 5 & 0 & 0 & 4 \\ 5 & 0 & 6 & 7 & 0 \\ 0 & 5 & 0 & 2 & 0 \\ 0 & 0 & 5 & 0 & 6 \\ 7 & 4 & 0 & 5 & 0\end{array}\right]$. The channel matrices are given as below:

$$
\begin{aligned}
& \boldsymbol{H}_{12}=\operatorname{diag}\{0,1\}, \boldsymbol{H}_{15}=\operatorname{diag}\{1,0\}, \boldsymbol{H}_{21}=\operatorname{diag}\{1,0\}, \\
& \boldsymbol{H}_{23}=\operatorname{diag}\{1,1\}, \boldsymbol{H}_{24}=\operatorname{diag}\{0,1\}, \boldsymbol{H}_{32}=\operatorname{diag}\{1,1\}, \\
& \boldsymbol{H}_{34}=\operatorname{diag}\{1,1\}, \boldsymbol{H}_{43}=\operatorname{diag}\{1,0\}, \boldsymbol{H}_{45}=\operatorname{diag}\{1,1\}, \\
& \boldsymbol{H}_{51}=\operatorname{diag}\{0,1\}, \boldsymbol{H}_{52}=\operatorname{diag}\{1,1\}, \boldsymbol{H}_{54}=\operatorname{diag}\{1,0\} .
\end{aligned}
$$

According to the definition of the channel Laplacian matrices, we can obtain them as follows:

$$
\begin{aligned}
\boldsymbol{R}_{1} & =\left[\begin{array}{ccccc}
-4 & 0 & 0 & 0 & 4 \\
5 & -11 & 6 & 0 & 0 \\
0 & 5 & -7 & 2 & 0 \\
0 & 0 & 5 & -11 & 6 \\
0 & 4 & 0 & 5 & -9
\end{array}\right] \\
\boldsymbol{R}_{2} & =\left[\begin{array}{ccccc}
-5 & 5 & 0 & 0 & 0 \\
0 & -13 & 6 & 7 & 0 \\
0 & 5 & -7 & 2 & 0 \\
0 & 0 & 0 & -6 & 6 \\
7 & 4 & 0 & 0 & -11
\end{array}\right]
\end{aligned}
$$

Let the nonlinear dynamics of the system be given by

$\boldsymbol{f}\left(\boldsymbol{x}_{i}, \boldsymbol{v}_{i}, t\right)=\left[\begin{array}{l}6 \sin (4 t)+0.01 \sin \left(2 x_{i 1}\right)+0.01 \cos \left(4 v_{i 1}\right) \\ 6 \sin (4 t)+0.01 \sin \left(2 x_{i 2}\right)+0.01 \cos \left(4 v_{i 2}\right)\end{array}\right]$

Combing with Assumption 1, one can obtain $l_{1}=$ $0.0008, l_{2}=0.0032$. Select $\beta=0.8, \varphi_{1}=0.00009, \varphi_{2}=$ $1.8, \gamma=0.1, \kappa=\sigma=500$ and $N_{M}=3$. Using MATLAB LMI Toolbox, we can obtain a feasible solution $\alpha=1.8031$ that satisfy the LMIs of Theorem 1. The cyber-physical power systems of the rotor angle and rotor speed are given by Figs. 4 and 5, respectively; one can find that all of the rotor angles and rotor speeds approach the common time-

Fig. 2 5-Generator 14-bus cyber physical power system 

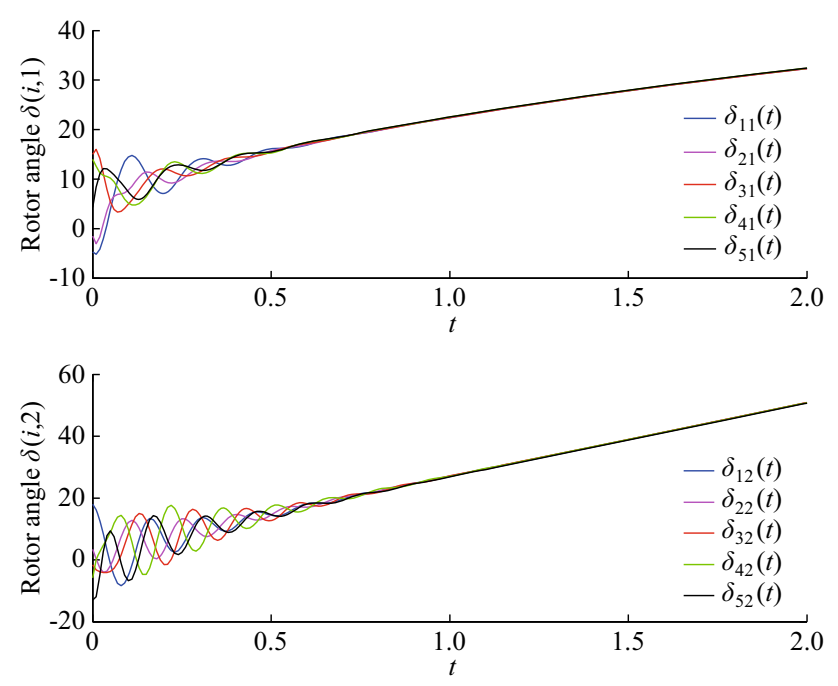

Fig. 4 Rotor angle of CPPS
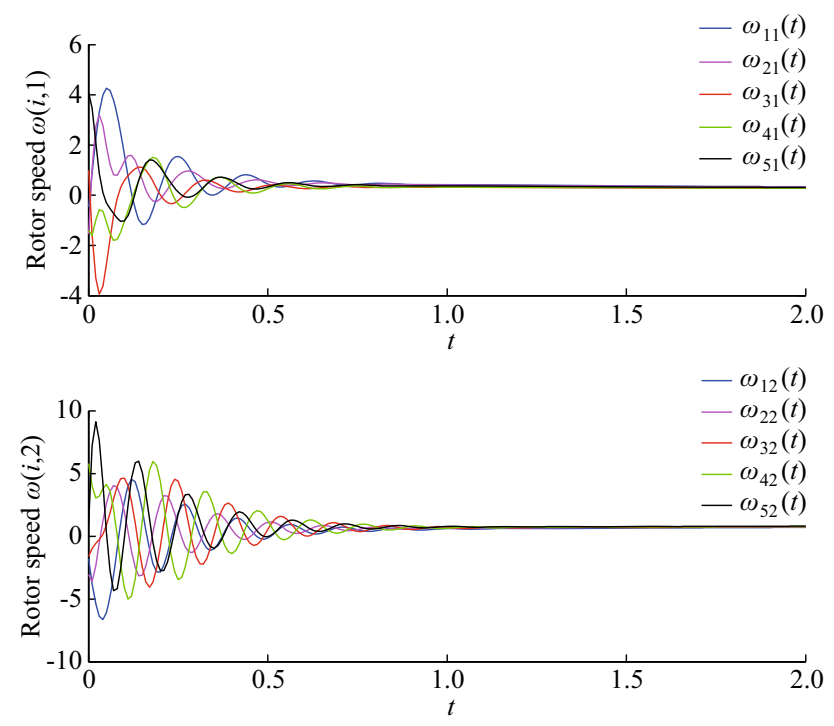

Fig. 5 Rotor speed of CPPS

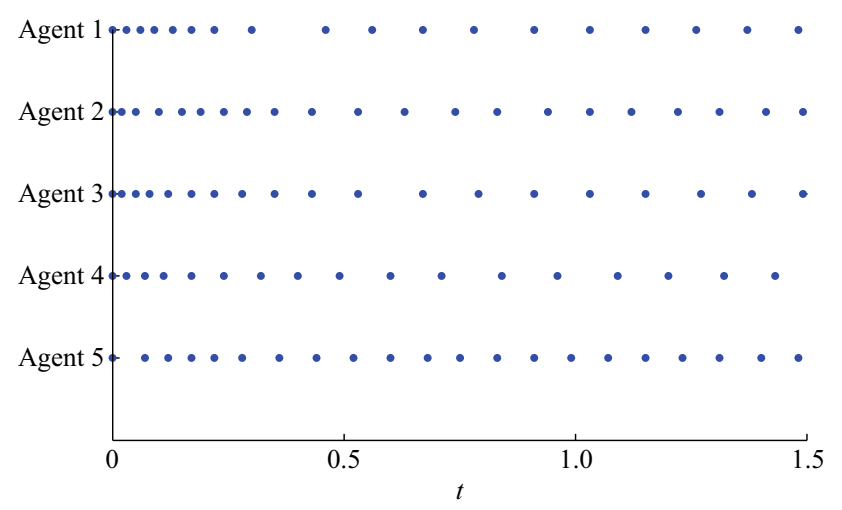

Fig. 6 Sampling instants of five agents varying value. Figure 6 shows the event-triggered sampling instants of all five agents of the system during the time period $[0,1.5]$.

\section{Conclusion}

Herein, the consensus of the CPPS based on the fractional-order MAS with communication constraints was investigated, and a sufficient consensus condition was derived. Owing to practical limitations, only partial information could be transmitted among agents. For the energy resource conservation, the event-trigger sampling strategy was considered and the Zeno behavior of the triggered time sequence was excluded. Thus, the proposed consensus protocol of the CPPS based on the fractional-order MAS model with communication constraints was more realistic and general. Therefore, the related consensus problem was challenging. Finally, a numerical example was presented to demonstrate the validity of the theoretical results.

Acknowledgements This work was jointly supported by the Research Project Supported by the Shanxi Scholarship Council of China (No. 2015044), the Fundamental Research Project of Shanxi Province (No. 2015021085), the National Science Foundation of China (No. 61603268, No. 61272530 and No. 61573096).

Open Access This article is distributed under the terms of the Creative Commons Attribution 4.0 International License (http:// creativecommons.org/licenses/by/4.0/), which permits unrestricted use, distribution, and reproduction in any medium, provided you give appropriate credit to the original author(s) and the source, provide a link to the Creative Commons license, and indicate if changes were made.

\section{References}

[1] Xie L, Ilic MD (2008) Module-based modeling of cyber-physical power systems. In: Proceedings of 28th international conference on distributed computing systems workshops, Beijing, China, 17-20 June 2008, 6 pp

[2] Zhao J, Wen F, Xue Y et al (2010) Cyber physical power systems: architecture, implementation techniques and challenges. Autom Electr Power Syst 34(16):1-7

[3] Zhao J, Wen F, Xue Y et al (2011) Modeling analysis and control research framework of cyber physical power systems. Autom Electr Power Syst 35(16):1-8

[4] Fallah YP, Huang C, Sengupta R et al (2010) Design of cooperative vehicle safety systems based on tight coupling of communication, computing and physical vehicle dynamics. In: Proceedings of 1st ACM/IEEE international conference on cyber-physical systems, Stockholm, Sweden, 13-15 April 2010, $9 \mathrm{pp}$

[5] $\mathrm{Hu} \mathrm{JB}, \mathrm{Li} \mathrm{F}, \mathrm{Wu} \mathrm{J}$ et al (2013) Research on aviation electric power cyber physical systems. Adv Mater Res 846-847:126-133

[6] Dobson I, Chiang HD (1989) Towards a theory of voltage collapse in electric power systems. Syst Control Lett 13(3):253-262 
[7] Wang HO, Abed E, Hamdan AM (1994) Bifurcations, chaos, and crises in voltage collapse of a model power system. IEEE Trans Circuits Syst I Fundam Theory Appl 41(4):294-302

[8] Hermanns H, Wiechmann H (2009) Future design challenges for electric energy supply. In: Proceedings of IEEE conference on emerging technologies and factory automation, Mallorca, Spain, 22-25 September 2009, 8 pp

[9] Vittal E, Keane A (2012) Rotor angle stability with high penetrations of wind generation. In: Proceedings of IEEE PES general meeting, San Diego, USA, 22-26 July 2012, 1 pp

[10] Li Y, Yong T, Cao J et al (2015) A consensus control strategy for dynamic power system look-ahead scheduling. Neurocomputing 168:1085-1093

[11] Cong L, Wang Y (2003) Co-ordinated control of generator excitation and statcom for rotor angle stability and voltage regulation enhancement of power systems. IEE Proc Gener Transm Distrib 149(6):659-666

[12] Ren W (2008) On consensus algorithms for double-integrator dynamics. IEEE Trans Autom Control 53(6):1503-1509

[13] Yu W, Chen G, Cao M (2010) Some necessary and sufficient conditions for second-order consensus in multi-agent dynamical systems. Automatica 46(6):1089-1095

[14] Fuentes-Fernndez R, Guijarro M, Pajares G (2009) A multiagent system architecture for sensor networks. Sensors 9(12):10244-10269

[15] Stipanovic DM, Inalhan G, Teo R et al (2004) Decentralized overlapping control of a formation of unmanned aerial vehicles. Automatica 40(8):1285-1296

[16] Wang W, Huang C, Cao J et al (2017) Event-triggered control for sampled-data cluster formation of multi-agent systems. Neurocomputing 267:25-35

[17] Huang S, Chu Y, Li S et al (2008) Application of multi-agent system in a microgrid. Autom Electr Power Syst 32(24):80-84

[18] Xu Y, Liu W, Gong J (2011) Stable multi-agent-based load shedding algorithm for power systems. IEEE Trans Power Syst 26(4):2006-2014

[19] Facchinetti T, Vedova MLD (2011) Real-time modeling for direct load control in cyber-physical power systems. IEEE Trans Ind Inform 7(4):689-698

[20] Wang Q, Tang Y, Li F et al (2016) Coordinated scheme of under-frequency load shedding with intelligent appliances in a cyber physical power system. Energies 9(8):1-14

[21] Wu Y, Rong L, Tang Y (2014) A distributed control method for power system rotor angle stability based on second-order consensus. In: Proceedings of 4 th annual international conference on cyber technology in automation, control, and intelligent systems, Hong Kong, China, 4-7 June 2014, 6 pp

[22] El-Khazali R, Memom Z, Tawalbeh N (2010) Fractional-order power system stabilizer. In: Proceedings of 4th IFAC workshop on fractional differentiation and applications, Badajoz, Spain, 18-20 October 2010, $6 \mathrm{pp}$

[23] Roohi M, Haghighi AR, Aghababa MP (2015) Stabilisation of unknown fractional-order chaotic systems: an adaptive switching control strategy with application to power systems. IET Gener Transm Distrib 9(14):1883-1893

[24] Chen L, Chai Y, Wu R et al (2012) Cluster synchronization in fractional-order complex dynamical networks. Phys Lett A 376(35):2381-2388

[25] Norelys A, Manuel A, Javier A (2014) Lyapunov functions for fractional order systems. Commun Nonlinear Sci Numer Simul 19(9):2951-2957

[26] Tavazoei MS, Haeri M (2009) A note on the stability of fractional order systems. Math Comput Simul 79(5):1566-1576

[27] Yin X, Yue D, Hu S (2013) Brief paper-consensus of fractionalorder heterogeneous multi-agent systems. IET Control Theory Appl 7(2):314-322
[28] Huang C, Ho D, Lu J (2015) Partial-information-based synchronization analysis for complex dynamical networks. J Frankl Inst 352(9):3458-3475

[29] Huang C, Ho D, Lu J (2012) Partial-information-based distributed filtering in two-targets tracking sensor networks. IEEE Trans Circuits Syst I Regul Pap 59(4):820-832

[30] Lu J, Ding C, Lou J et al (2015) Outer synchronization of partially coupled dynamical networks via pinning impulsive controllers. J Frankl Inst 352(11):5024-5041

[31] Fan Y, Liu L, Feng G et al (2015) Self-triggered consensus for multi-agent systems with zeno-free triggers. IEEE Trans Autom Control 60(10):2779-2784

[32] Zhang W, Tang Y, Liu Y et al (2017) Event-triggering containment control for a class of multi-agent networks with fixed and switching topologies. IEEE Trans Circuits Syst I Regul Pap 64(3):619-629

[33] Li L, Ho D, Lu J (2017) Event-based network consensus with communication delays. Nonlinear Dyn 87(3):1847-1858

[34] Fan Y, Yang Y, Zhang Y (2016) Sampling-based event-triggered consensus for multi-agent systems. Neurocomputing 191:141-147

[35] Noorbakhsh SM, Ghaisari J (2016) Distributed event-triggered consensus strategy for multi-agent systems under limited resources. Int J Control 89(1):156-168

[36] Dimarogonas D, Frazzoli E, Johansson K (2012) Distributed event-triggered control for multi-agent systems. IEEE Trans Autom Control 57(5):1291-1297

[37] Li H, Liao X, Huang T et al (2015) Event-triggering sampling based leader following consensus in second-order multi-agent systems. IEEE Trans Autom Control 60(7):1998-2003

[38] Yang D, Ren W, Liu X et al (2016) Decentralized event-triggered consensus for linear multi-agent systems under general directed graphs. Automatic 69:242-249

[39] Zhou F, Huang Z, Liu W et al (2014) An event-triggered consensus control with sampled-data mechanism for multi-agent systems. Int Fed Autom Control 47(3):6959-6964

[40] Lc P, Dw H (2007) Remarks on fractional derivatives. Appl Math Comput 187(2):777-784

[41] Tuglie E, Iannone S, Torelli F (2008) A coherency-based method to increase dynamic security in power systems. Electr Power Syst Res 78(8):1425-1436

[42] Lu J, Wang Z, Cao J et al (2012) Pinning impulsive stabilization of nonlinear dynamical networks with time-varying delay. Int $\mathrm{J}$ Bifurc Chaos 22(7):1250176

[43] Lu W, Chen T (2006) New approach to synchronization analysis of linearly coupled ordinary differential systems. Phys D Nonlinear Phenom 213(2):214-230

[44] Podlubny I (1997) The Laplace transform method for linear differential equations of the fractional order. https://arxiv.org/ pdf/funct-an/9710005.pdf. Accessed 30 October 1997

[45] Li H, Chen G, Dong Z et al (2016) Consensus analysis of multiagent systems with second-order nonlinear dynamics and general directed topology: an event-triggered scheme. Inf Sci 370-371:598-622

[46] Raberto M, Scalas E, Mainardi F (2002) Waiting-times and returns in high-frequency financial data: an empirical study. Phys A Stat Mech Its Appl 314(1-4):749-755

[47] Wang F, Yang Y (2017) Leader-following consensus of nonlinear fractional-order multi-agent systems via event-triggered control. Int J Syst Sci 48(3):571-577

Chi HUANG received the M.S. degree in mathematics and the Ph.D. degree with the Department of Mathematics from the City University of Hong Kong, Hong Kong, China, in 2009 and 2012, respectively. He was the recipient of the Best Paper Award in the Eighth Asian 
Control Conference in 2011. His current research interests include multi-agent systems, complex dynamical networks, and sensor networks.

Chengli FENG received the B.S. degree in mathematics from Taiyuan Normal University, Taiyuan, China, in 2014. She is now a M.S. candidate at Taiyuan University of Technology. Her main research interests include synchronization of complex networks and consensus of multi-agent systems.

Jinde CAO is a Distinguished Professor, the Dean of School of Mathematics and the Director of the Research Center for Complex Systems and Network Sciences at Southeast University. From March 1989 to May 2000, he was with the Yunnan University. In May 2000, he joined the Department of Mathematics, Southeast University, Nanjing, China. From July 2001 to June 2002, he was a Postdoctoral Research Fellow at the Department of Automation and ComputerAided Engineering, Chinese University of Hong Kong, Hong Kong, China. He was an Associate Editor of the IEEE Transactions on Neural Networks, Journal of the Franklin Institute, Neurocomputing, and Differential Equations and Dynamical Systems. He is an Associate Editor of the IEEE Transactions on Cybernetics, IEEE Transactions on Cognitive and Developmental Systems, Advances in Difference Equations, Mathematics and Computers in Simulation, and Neural Networks. He is a Fellow of IEEE, and a Member of the Academy of Europe. He has been named as Highly-Cited Researcher in Mathematics, Computer Science and Engineering by Thomson Reuters. 